\title{
Recent progress in pressure and temperature tactile sensors: principle, classification, integration and outlook
}

\author{
Jiajie Yu ${ }^{1, \#}, K^{\prime}$ Zhang ${ }^{1, \#}$, Yuan Deng ${ }^{1,2}$ \\ ${ }^{1}$ Hangzhou Innovation Center, Beihang University, Hangzhou 310052, Zhejiang, China. \\ ${ }^{2}$ Research Institute for Frontier Science, Beihang University, Beijing 100083, China. \\ \#Authors contributed equally.
}

Correspondence to: Prof. Yuan Deng, Hangzhou Innovation Center, Beihang University, No.18 Chuanghui Street, Hangzhou 310052, Zhejiang, China. E-mail: dengyuan@buaa.edu.cn

How to cite this article: Yu J, Zhang K, Deng Y. Recent progress in pressure and temperature tactile sensors: principle, classification, integration and outlook. Soft Sci 2021;1:6. https://dx.doi.org/10.20517/ss.2021.05

Received: 21 Jun 2021 First Decision: 20 Jul 2021 Revised: 4 Aug 2021 Accepted: 12 Aug 2021 First online: 12 Aug 2021

Academic Editor: Zhifeng Ren Copy Editor: Yue-Yue Zhang Production Editor: Yue-Yue Zhang

\begin{abstract}
Tactile sensors have received increasing research interest owing to the broad applications in areas of health monitoring, artificial intelligence, robotics, and prosthetics. The ability to understand and perceive touch and heat is of importance because it helps people to recognize objects, prevent injury, and provide heat information from grasped objects. However, bimodal tactile sensors often suffer from signal interference and complicated fabrication process. Numerous efforts have been undertaken to develop highly independent sensors based on different transduction principles as well as the device integration techniques. Here, strategies for improvement of main performance parameters such as sensitivity, sensing range, hysteresis, response/recovery time, and stability are discussed. A comprehensive overview of important progress in pressure and temperature tactile sensors in recent years is summarized. According to sensor units and transduction principles, temperature and pressure tactile sensors are categorized into two types: dual-parameter sensors and integrated bimodal sensors. Integration of tactile sensors from the viewpoint of power supply, wireless communication, and signal process circuit is given. Finally, challenges and outlook are provided and presented for pressure and temperature tactile sensors.
\end{abstract}

Keywords: Tactile sensor, pressure sensor, temperature sensor, dual-parameter sensor, bimodal sensor adaptation, distribution and reproduction in any medium or format, for any purpose, even commercially, as long as you give appropriate credit to the original author(s) and the source, provide a link to the Creative Commons license, and indicate if changes were made. 


\section{INTRODUCTION}

Humans have five basic senses: visual, auditory, tactile, gustatory, and olfactory. Tactile, one of the most important sensing modalities, allows us to identify object hardness and shapes, distinguish material textures, and perceive temperature and pains. Tactile is perceived by skin cells, which directly send signals through the nerves to the central nervous systems, where they are transformed into electrical signals and interpreted. Tactile sensing is usually related to the measurement of spatial distribution of various signals including force (pressure, strain, slip, friction, and shear), heat (temperature and thermal conductivity), and humidity.

Tactile sensors, capable of sensing the location and surface properties of objects, have attracted considerable attention due to the diverse applications in health monitoring, artificial intelligence, robotics, and prosthetics ${ }^{[1-4]}$. Despite these advances, great challenges remain in multifunctional tactile sensors that can simultaneously detect two or more external stimuli without noticeable interferences. One challenge is that integrating multiple sensors for multimodal stimuli often requires complicated device structure and fabrication process ${ }^{[5-7]}$. Therefore, many efforts have been undertaken to develop highly independent sensors based on different transduction principles as well as device integration methods. The other challenge is that the crosstalk effect from parasitic parallel paths will appear when presented in an array form. Thus, various scanning approaches and readout circuits have been developed and investigated to reduce this phenomenon.

The ability to understand and perceive touch and heat is of importance because it helps people to recognize objects, to prevent injury and provide heat information from grasped objects ${ }^{[8]}$. Except sensing of touch and heat, that of humidity is also a critical part for mimicking human skin. This topic will be addressed in a separate paper. Pressure stimuli detection methods based on piezoresistivity ${ }^{[9,10]}$, capacitance ${ }^{[1,12]}$, piezoelectricity ${ }^{[1,1,1]}$, and triboelectricity ${ }^{[15,16]}$ transduction principles have been extensively studied in previous literature. For temperature sensing, thermoresistive- ${ }^{[17,18]}$ and thermoelectric-based ${ }^{[1,20]}$ temperature sensors have been widely employed. Strategies for improvement of some main performance parameters are discussed in Section "Performance parameters evaluation of tactile sensors: sensitivity, sensing range, hysteresis, and response/recovery time". To fully mimic human skins, bimodal flexible sensors for simultaneous quantitative detection of pressure and temperature are highly desired. Recent advances in pressure and temperature sensors are presented in this review, and they have been categorized into two types according to sensor units and transduction principles: (1) dual-parameter sensors (one individual sensor) that can respond to pressure/temperature stimuli as separated signals by one individual; and (2) integrated bimodal sensors (two individual sensors integrated into one pixel) where each responds to one specific stimuli. In Section "CLASSIFICATION OF PRESSURE AND TEMPERATURE TACTILE SENSORS”, we discuss them in detail.

With the explosion of the Internet of Things, an ideal pressure and temperature tactile sensor often requires high power supply techniques and low power wireless communication systems. Recent energy harvesting and storage techniques such as photovoltaics, thermoelectrics, piezoelectrics, and triboelectrics are highlighted in Section "Power supply: energy harvesting and energy storage techniques". Wireless communication protocols including NFC, BLE, ZigBee, and Wi-Fi are presented in Section "wireless communication: NFC, BLE, ZigBee, and Wi-Fi". Due to the crosstalk effect resulting from parasitic parallel paths in an array form, some typical scanning approaches are introduced in Section "Signal process circuits".

Herein, we aim to present a literature review on the recent progress in pressure and temperature tactile sensors. The main contents include the following aspects: (1) basic concepts, transduction principles, and 
performance parameters of pressure and temperature tactile sensors; (2) classification of pressure and temperature tactile sensors; (3) integration of tactile sensors in terms of power supply, wireless communication, and signal process circuit; and (4) current challenge and future outlook for pressure and temperature tactile sensors.

\section{FUNDAMENTALS ABOUT TACTILE SENSOR: TRANSDUCTION PRINCIPLES AND PERFORMANCE PARAMETERS}

The definition of tactile sensor was originally given by Lee and Nicholls ${ }^{[21]}$ : a device or system that can measure a given property of an object or contact event through physical contact between the sensor and the object. In this section, basic working principles and performance parameters of tactile sensor are summarized. We mainly focus on the description of tactile sensor that is capable of detecting both pressure and temperature signals. A schematic illustration of the recent advances in pressure and temperature tactile sensors in terms of principles, classification, and integration is displayed in Figure 1.

\section{Transduction principles of tactile sensors: pressure and temperature sensing}

Pressure sensing mechanisms: piezoresistivity, capacitance, piezoelectricity, and triboelectricity

Pressure sensors have attracted intense attention due to the wide applications in areas of human motion detection, intelligent robotics, and artificial prosthetics. There are several transduction methods for pressure stimuli detection including piezoresistivity, capacitance, piezoelectricity, and triboelectricity. The advantages and disadvantages of the major pressure sensor types are listed in Table 1. Piezoresisitive pressure sensors detect pressure stimuli through the change in electric resistance of the device and have been investigated extensively due to their simple design and readout mechanism. Sundaram et al..$^{[9]}$ demonstrated a tactile glove with scalable deep convolutional neural networks and prepared commercial force sensing resistors called velostat, which could identify and estimate the weight of objects. Capacitive pressure sensors are mainly composed of two parallel conductive plates separated by a dielectric material. The major advantages of capacitive pressure sensors are the simple governing principle and temperature independence. However, sensitivity and signal-to-noise ratio are reduced for miniaturization. Lee et al. ${ }^{[22]}$ demonstrated a capacitive pressure sensor fabricated by composite nanofibers of CNTs and graphene as sensing materials. The bending insensitivity was achieved by changing the relative alignment of nanofibers during deformation. Piezoelectric pressure sensors are based on the mechanism that electric dipole moments are generated in anisotropic crystalline materials when mechanical stimuli are applied. Unlike piezoresisitive and capacitive pressure sensors, being capable of static and dynamic measurements, piezoelectric sensors are especially suitable for dynamic pressure measurements ${ }^{[14]}$. Furthermore, piezoelectric pressure sensors illustrate an impressive property of self-powering ability, making it practicable to the associated circuit system. Chen et al. ${ }^{[13]}$ developed a $\mathrm{P}(\mathrm{VDF}-\mathrm{TrFE})$ micropillar-based piezoelectric tactile sensor array integrated by a portable signal processing circuit, showing an impressive self-powering ability for dynamic tactile sensing. Triboelectric pressure sensors were originally demonstrated by Wu et al. ${ }^{[23]}$ in 2012 . In brief, triboelectrification generates oppositely polarized charges on contacted material surfaces, while electrostatic induction is attributed to the conversion of mechanical energy into electric power by mechanically agitated separation.

\section{Temperature sensing mechanisms: thermoresistive and thermoelectric effects}

Apart from pressure stimuli, sensing temperature is another important functionality of tactile sensor to prevent injury and provide critical heat information about the grasped object. Generally, a temperature resolution as small as $0.02{ }^{\circ} \mathrm{C}$ is required for tactile sensors. The advantage of a flexible temperature sensor array is that the flexible nature allows the sensor array to be blended and twisted, allowing greater freedom in design and operation. In addition, flexible temperature sensor arrays will reduce many types of wiring, 
Table 1. Advantages and disadvantages of major pressure sensor types

\begin{tabular}{lll}
\hline Type & Advantages & Disadvantages \\
\hline Piezoresistivity & High spatial resolution, low cost, simple manufacturing & Non-linear response, hysteresis, fatigue \\
Capacitance & High sensitivity, high spatial resolution, temperature independence & Susceptibility to noise, crosstalk, complexity \\
Piezoelectricity & Self-powering, high sensitivity & Temperature dependence, dynamic sensing only \\
Triboelectricity & Self-powering, high sensitivity & Temperature dependence, dynamic sensing only \\
\hline
\end{tabular}

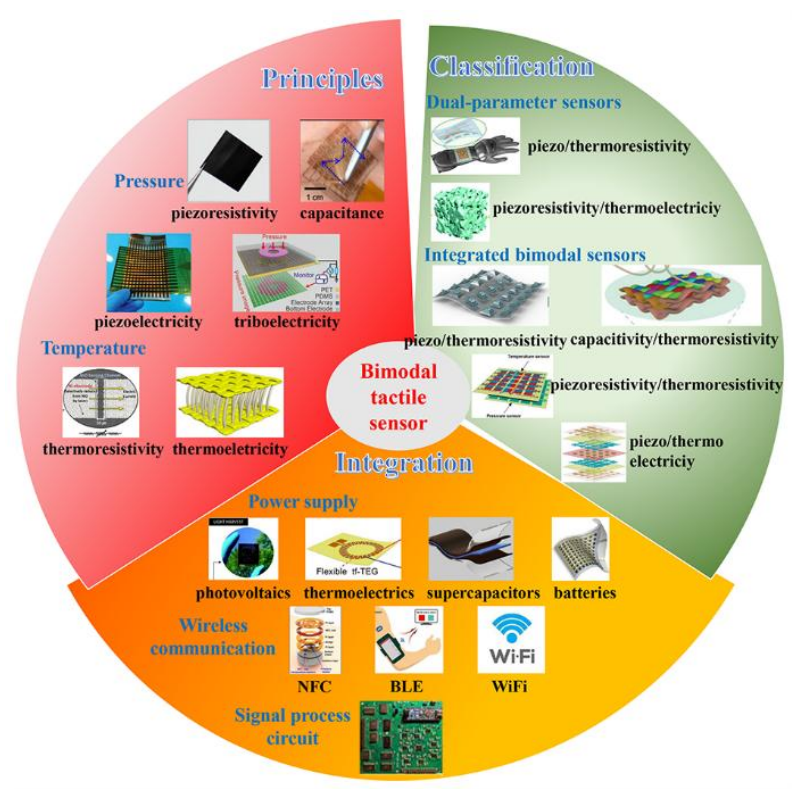

Figure 1. Overview of recent progress in pressure and temperature tactile sensors in terms of principles, classification, and integration. Figure "piezoresistivity", quoted with permission from Sundaram et al. ${ }^{[9]}$; "capacitance", quoted with permission from Park et al. ${ }^{[11]}$; "piezoelectricity", quoted with permission from Chen et al. ${ }^{[13]}$; "triboelectricity", quoted with permission from Wang et al. ${ }^{[15]}$; "thermoresistivity", quoted with permission from Shin et al. ${ }^{[17]}$; "thermoelectricity", quoted with permission from Li et al. ${ }^{[19] ;}$ "piezo/thermoresistivity" (dual-parameter sensors), quoted with permission from Wang et al. .30],"piezoresistivity/thermoelectricity"

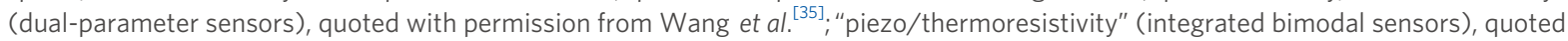
with permission from Jung et al. ${ }^{[37], " c a p a c i t i v i t y / t h e r m o r e s i s t i v i t y ", ~ q u o t e d ~ w i t h ~ p e r m i s s i o n ~ f r o m ~ W u ~ e t ~ a l . ~}{ }^{[40]}$; "piezoresistivity/thermoelectricity" (integrated bimodal sensors), quoted with permission from Jung etal. ${ }^{[42]}$; "piezo/thermoelectricity", quoted with permission from Zhu et al. ${ }^{[46]}$; "photovoltaics", quoted with permission from Michaels et al. ${ }^{[50]}$; "thermoelectrics", quoted with permission from Michaels et al. ${ }^{[50]}$; "supercapacitors", quoted with permission from Wang et al. ${ }^{[61]}$ ', "batteries", quoted with permission from Pan et al. ${ }^{[59], ~ " N F C ", ~ q u o t e d ~ w i t h ~ p e r m i s s i o n ~ f r o m ~ H a n ~ e t ~ a l . ~}{ }^{[62]}$; "BLE", quoted with permission from Song et al. ${ }^{[63]}$; "Signal process circuit", quoted with permission from Luo et al. ${ }^{[64]}$.

thus reducing the circuit complexity and wiring cost. However, flexible temperature sensor arrays also suffer the problem that not all manufacturers are equipped to provide flexible electronic products suitable for the FPC to their customers. Numerous methods have been achieved to detect temperature, including thermoresistive resistors, thermoelectric sensors, infrared sensors, and optical sensors. Among them, two typical classes of temperature sensing mechanism based on thermoresistive and thermoelectric effects are the most commonly used in flexible tactile sensors. Thermoresistive temperature sensors are based on the principle that electric resistance varies with temperature because of the change in electric conductivity, usually quantified by temperature coefficient of resistance. In a recent work reported by Shin et al. ${ }^{[17]}$, a thermoresistive $\mathrm{NiO}$ semiconductor temperature sensor was prepared by coating $\mathrm{NiO}$ nanoparticles ink on PET by doctor blading technique, followed by monolithic selective laser reductive sintering $\mathrm{NiO}$ into Ni. In addition to the thermoresistive effect, thermoelectric temperature sensors are based on the thermoelectric effect which allows the transformation of heat into electricity. The generated voltage $(\Delta V)$ can be 
determined by the following equation: $V=S \times \Delta T$, where $S$ represents for the Seebeck coefficient and $\Delta T$ means the temperature difference. The main advantage of thermoelectric temperature sensors is that they do not require an external power supply. Liu et al. ${ }^{[24]}$ reported a thermocouple consisting of n-type $\mathrm{La}_{0.8} \mathrm{Sr}_{0.2}$ $\mathrm{CrO}_{3}$ and p-type $\mathrm{In}_{2} \mathrm{O}_{3}$ with a giant thermoelectric voltage of $410.3 \mathrm{mV}$ at $1270{ }^{\circ} \mathrm{C}$.

\section{Performance parameters evaluation of tactile sensors: sensitivity, sensing range, hysteresis, response/recovery time, stability, and repeatability}

The key parameters that evaluate the performance of tactile sensors are sensitivity, sensing range, hysteresis, response/recovery time, and temperature coefficient. Strategies for improvement of these parameters for tactile sensors are given below.

\section{Sensitivity}

Sensitivity is an important parameter for tactile sensors, quantified by the slope of electrical response $v s$. tactile stimuli. A higher sensitivity means better signal-to-noise ratio under external stimuli, allowing subtle changes to be detected. There are two methods to improve sensitivity of piezoresistive and capacitive-type pressure sensors: (1) porous structures; and (2) periodic microstructured array. Additionally, sensitivity of capacitive pressure sensors can also be improved by being integrated into active transistor matrix. One approach for piezoelectric pressure sensors relies on the employment of materials possessing intrinsic giant piezoelectric effect, such as semiconducting $\mathrm{ZnO}$ nanowires. The techniques of improvement in temperature sensitivity of thermoresistive conductive polymers are similar to that of piezoresistive materials. In addition, the sensitivity of thermoresistive temperature sensors can also be enhanced by the design of serpentine trace, thus increasing the relative resistance change. Shi et al ${ }^{[25]}$ demonstrated F-N tunneling effect-based pressure sensors, which achieved the high sensitivity of $260.3 \mathrm{kPa}^{-1}$. The pressure sensor was fabricated by spin coating sea urchin such as carbon spheres in PDMS [Figure 2]. The quantum effect mechanism to enhance sensitivity is quite different from previously reported methods.

\section{Sensing range}

Sensing range, also called dynamic range, depends on the exact application. An ideal tactile sensor should possess high sensitivity over a broad sensing range ${ }^{[26]}$. Most pressure sensors cannot satisfy the needs of both high sensitivity and large sensing range. One efficient approach to realize wide sensing range without obviously reduced sensitivity is to create hierarchical structure array. Bae et al. ${ }^{[27]}$ introduced a hierarchical structure array pressure sensor to achieve high sensitivity of $8.5 \mathrm{kPa}^{-1}$ in a broad pressure range up to $12 \mathrm{kPa}$ with excellent linearity, as shown in Figure 3. Polymeric temperature sensors usually suffer from a narrow temperature sensing range; copolymers using two specific monomers in different mixture ratios are preferable to widen sensing range.

\section{Hysteresis}

Hysteresis is the difference between two separate signal measurements when the same tactile stimuli are applied. It is a critical parameter in tactile sensors and cannot be ignored for practical applications. Measurements of hysteresis depend on the increasing or decreasing rate of tactile stimuli and number of cycles the tactile sensor has previously experienced, thus making it difficult to obtain an accurate value. That means there is no standardization in obtaining hysteresis. Especially for piezoresistive sensors composed of conductive polymer composites, permanent resistance change will occur due to the slippage between the conductive filler and viscous polymer. One efficient approach to reduce hysteresis is to lower the viscous properties of the sensing materials and strengthen the bonding force. 


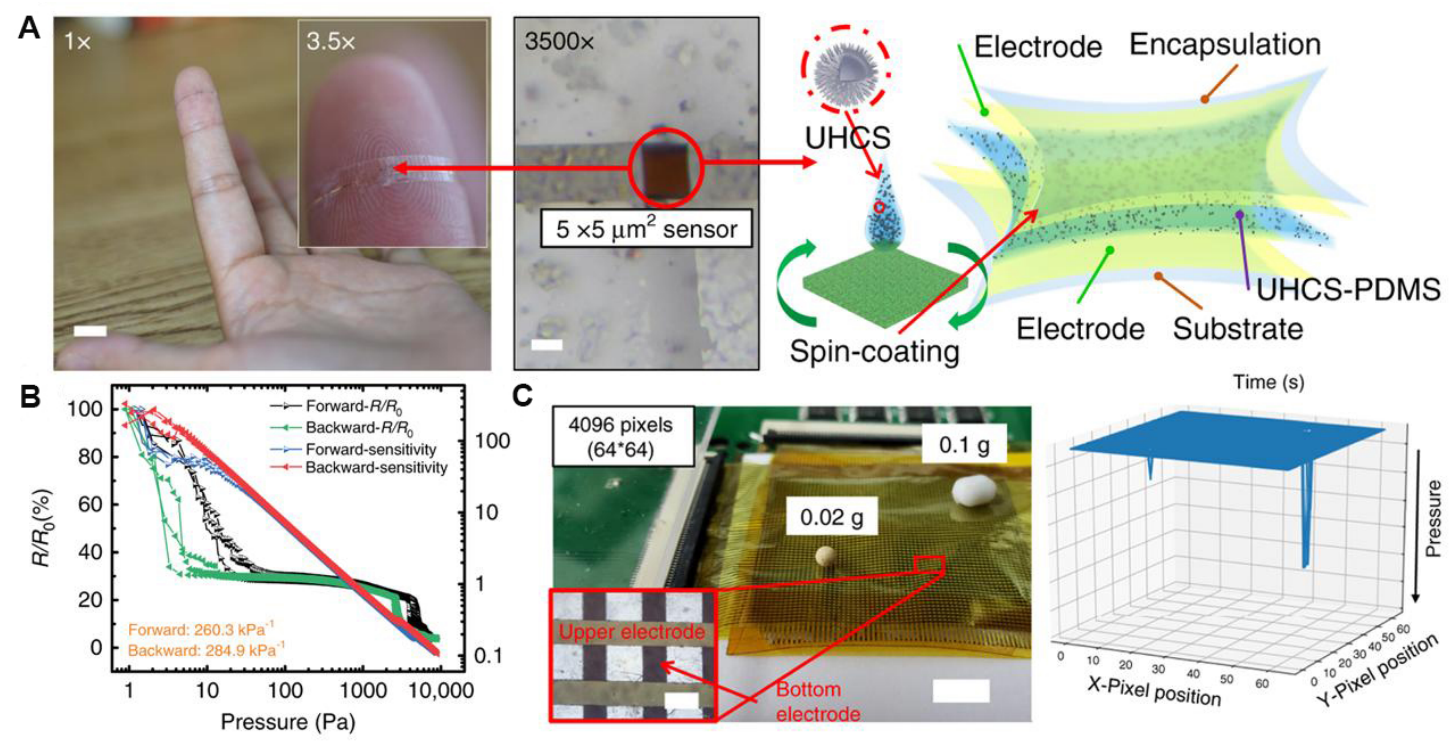

Figure 2. (A) Optical photograph and design illustration of ultrahigh sensitive pressure sensor; (B) resistance response and pressure sensitivity of the sensor; and (C) pressure detection of two tiny balls by a $64 \times 64$ pixel pressure sensor. This figure is quoted with permission from Shi et al. ${ }^{[25]}$.

A

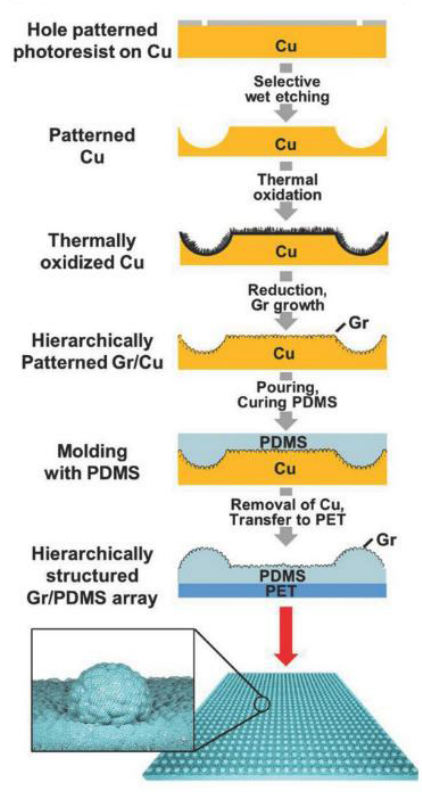

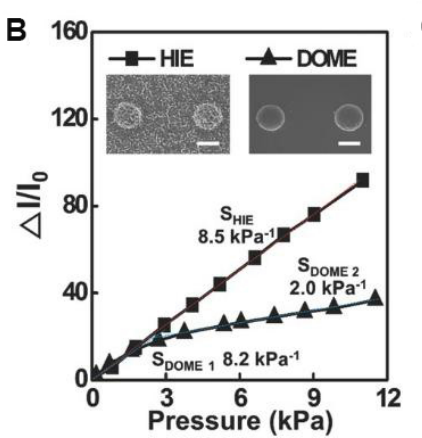

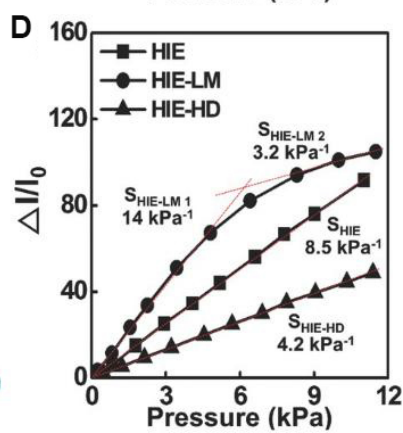

C

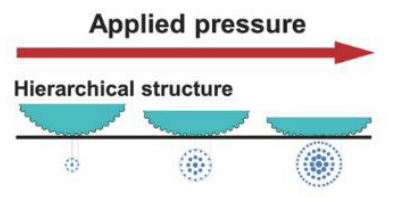

Dome structure

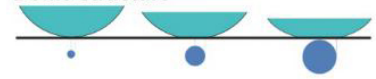

E

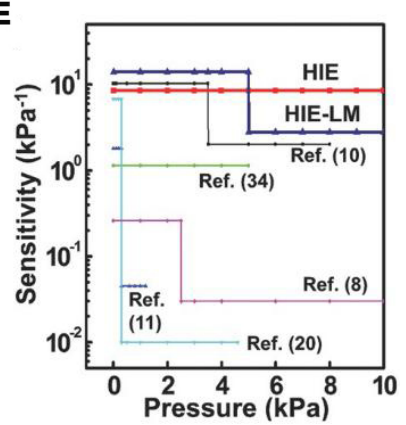

Figure 3. (A) Illustration of the ressure sensor fabrication process; (B) pressure response shows the effects of geometrical structure; (C) illustration of hierarchical structure effects under applied pressure; (D) influences of different parameters on sensitivity and pressure range; and (E) comparison with other pressure sensors. This figure is quoted with permission from Bae et al. ${ }^{[27]}$.

\section{Response/recovery time}

Response/recovery time is the time taken for a tactile sensor to reach $90 \%$ of the equilibrium point when stimuli is being applied and released. Similar to hysteresis, there is no standard method to attain response/recovery time. The response/recovery time is associated with the viscoelasticity property of 
elastomer, which can be reduced when the elasticity of the elastomer is enhanced. Various microstructures such as pyramidal, dome, and hemispheric have been employed to reduce response/recovery time, with pyramidal microstructures originally reported in the pioneering work of Chou et al ${ }^{[28]}$.

\section{Stability and repeatability}

In addition to sensitivity, sensing range, and linearity, the stability and repeatability of tactile sensors are important for long-term use. The quality, homogeneity, and consistency of raw materials will depend on the stability and repeatability characteristics, which have proven to be the bottleneck for practical applications. In addition to the raw materials, device processability is another challenge, vital for the device stability. The device interface quality and uniformity will affect the performance of the sensor device.

Comparison of the performance parameters of typical works are summarized in Table 2.

\section{CLASSIFICATION OF PRESSURE AND TEMPERATURE BIMODAL TACTILE SENSORS}

To fully mimic human skins, tactile sensors for simultaneous quantitative detection of pressure and temperature signals are highly desired. According to sensor units and transduction principle, pressure and temperature sensors are categorized into two types: (1) dual-parameter sensors; and (2) integrated bimodal sensors. A dual-parameter sensor refers to one individual sensor that can transduce pressure and temperature bimodal stimuli into separated signals. An integrated bimodal sensor is defined as two individual sensors integrated into one pixel that can transduce pressure and temperature bimodal stimuli into separated signals. In the following, we discuss and present recent examples of these tactile sensors in detail.

\section{Dual-parameter sensors}

One simple approach to achieve a pressure and temperature bimodal sensor is to develop a dual-parameter sensor that can respond to pressure/temperature stimuli as separated signals by one individual sensor. The major advantage of this class of tactile sensors is the facile fabrication process and readout mechanism. Two types of piezoresistive/thermoresistive and piezoresistive/thermoelectric have been widely reported in previous literature. Only one active material is used for sensing pressure and temperature signals.

\section{Piezoresistive/thermoresistive-type dual-parameter sensors}

It is obvious that this type of dual-parameter sensor usually detects piezoresistive/thermoresistive signals. Zhao et al. ${ }^{[29]}$ fabricated a flexible pressure and temperature dual-parameter sensor based on a MOF-derived $\mathrm{PC} / \mathrm{PDMS}$ composite. The piezoresistive and thermoresistive properties of the active sensing materials enable the as-prepared sensor to perform pressure and temperature sensing. Wang et al.$^{[30]}$ presented an all silk-derived temperature and pressure dual-parameter E-skin sensor, which was assembled through pressure and temperature sensors by lamination [Figure 4]. The as-prepared dual-parameter sensor could distinguish pressure and temperature signals under external stimuli such as exhaling and finger touching. $\mathrm{Zu}$ et al.$^{[31]}$ reported a pressure and temperature dual-parameter sensor, which used superhydrophobic aerogels as the piezoresistive/thermoresistive materials. The aerogel-based sensing array was capable of detecting temperature in the range of $20-100{ }^{\circ} \mathrm{C}$ and pressure in the range of $10 \mathrm{~Pa}$ to $110 \mathrm{kPa}$. However, due to the simplicity of the transduction mechanism, signal interference often occurs in decoupling analysis, which causes discrimination between pressure and temperature signal; this remains a great challenge.

\section{Piezoresistive/thermoelectric-type dual-parameter sensors}

Unlike the first class of dual-parameter sensor, this type of sensor usually detects piezoresistive/thermoelectric signals. In their pioneering work, Zhang et al.$^{[32]}$ introduced a flexible pressure 
Table 2. Comparison of the performance parameters of typical works

\begin{tabular}{|c|c|c|c|}
\hline Typical work & Sensitivity & Response/recovery time & Stability and repeatability \\
\hline Zhao et al. ${ }^{[29]}$ & $\begin{array}{l}\mathrm{P}: 15.63 \mathrm{kPa}^{-1} \\
\mathrm{~T}: 0.11^{\circ} \mathrm{C}^{-1}\end{array}$ & $\begin{array}{l}\mathrm{P}:<65 \mathrm{~ms} \\
\mathrm{~T}: \sim 100 \mathrm{~ms}\end{array}$ & P: $\sim 2000$ cycles \\
\hline Wang et al. ${ }^{[30]}$ & $\begin{array}{l}\text { P: } 3.01 \mathrm{kPa}^{-1} \\
\mathrm{~T}: 0.81^{\circ} \mathrm{C}^{-1}\end{array}$ & $\mathrm{~T}: 1800 \mathrm{~ms}$ & \\
\hline Zhang et al. ${ }^{[32]}$ & $\begin{array}{l}\mathrm{P}: 28.9 \mathrm{kPa}^{-1} \\
\mathrm{~T}: 1^{\circ} \mathrm{C}^{-1}\end{array}$ & $\begin{array}{l}\mathrm{P}:<20 \mathrm{~ms} \\
\mathrm{~T}:<2000 \mathrm{~ms}\end{array}$ & P: 10,000 cycles \\
\hline Wang et al. ${ }^{[34]}$ & $\begin{array}{l}\text { P: } 15.22 \mathrm{kPa}^{-1} \\
\mathrm{~T}: 1^{\circ} \mathrm{C}^{-1}\end{array}$ & $\mathrm{P}:<74 \mathrm{~ms}$ & P: 3000 cycles \\
\hline Wang et al. ${ }^{[35]}$ & $\begin{array}{l}\text { P: } 0.92 \mathrm{kPa}^{-1} \\
\mathrm{~T}: 17.1 \mathrm{mV}^{\circ} \mathrm{C}^{-1}\end{array}$ & P: $44 \mathrm{~ms}$ & P: 10,000 cycles \\
\hline Jung et al. ${ }^{[37]}$ & $\mathrm{T}: 0.98^{\circ} \mathrm{C}^{-1}$ & $P: 120 \mathrm{~ms}$ & P: 10,000 cycles \\
\hline Wang et al. ${ }^{[38]}$ & $P: 1185.8 \mathrm{kPa}^{-1}$ & P: $44 \mathrm{~ms}$ & P: 5000 cycles \\
\hline Bae et al. ${ }^{[39]}$ & $\begin{array}{l}\mathrm{P}: 0.7 \mathrm{kPa}^{-1} \\
\mathrm{~T}: 0.83 \%{ }^{\circ} \mathrm{C}^{-1}\end{array}$ & $\begin{array}{l}\mathrm{P}: 50 \mathrm{~ms} \\
\mathrm{~T}: 100 \mathrm{~ms}\end{array}$ & \\
\hline Wu et al. ${ }^{[40]}$ & $\begin{array}{l}\text { P: } 0.136 \mathrm{kPa}^{-1} \\
\mathrm{~T}: 1.23^{\circ} \mathrm{C}^{-1}\end{array}$ & P: $250 \mathrm{~ms}$ & P: 5000 cycles \\
\hline Shin et al. ${ }^{[41]}$ & $\mathrm{T}: 13.6 \mu \mathrm{V}^{\circ} \mathrm{C}^{-1}$ & P: $40 \mathrm{~ms}$ & P: 10,000 cycles \\
\hline Jung et al. ${ }^{[42]}$ & $\begin{array}{l}\text { P: } 0.64 \mathrm{VkPa}^{-1} \\
\text { T: } 109.4 \mu \mathrm{V}^{\circ} \mathrm{C}^{-1}\end{array}$ & $\mathrm{~T}: 37 \mathrm{~ms}$ & P: 3000 cycles \\
\hline
\end{tabular}

P: Pressure; T: temperature.

and temperature dual-parameter sensor constructed by PEDOT:PSS coated PU. Pressure and temperature signals were distinguished owing to different transduction piezoresistive and thermoelectric principles. The as-prepared dual-parameter tactile sensor exhibits a pressure sensitivity of larger than $28.9 \mathrm{kPa}^{-1}$ and a temperature resolution of less than $0.1 \mathrm{~K}$. In addition, this tactile sensor can be self-powered due to thermoelectric generation. Han et al ${ }^{[33]}$ demonstrated a temperature and pressure dual-parameter sensor based on organic PNG aerogels treated with DMSO vapor. The pressure and temperature signals were decoupled because of the temperature independent DMSO treated aerogels display semiconductor to semimetal transition, thus the crosstalk effect can be avoided. Recently, Wang et al. ${ }^{[34]}$ also reported a pressure and temperature dual-parameter sensor composed of hydrophobic films and graphene/PDMS sponges. For pressure sensing, the obtained pressure sensor shows a sensitivity of $>15.22 \mathrm{kPa}^{-1}$ with stability over $>3000$ cycles via piezoresistive effect. For temperature detection, the thermoelectric temperature sensor displays a temperature resolution of $1 \mathrm{~K}$. Wang et al. ${ }^{[35]}$ also proposed a pressure and temperature dual-parameter tactile sensor using organic thermoelectric PANI/CNTs decorated porous PDMS as active sensing materials [Figure 5]. The as-prepared sensor can detect static/dynamic pressure and temperature signals with a pressure sensitivity of $0.918 \mathrm{kPa}^{-1}$ up to $130 \mathrm{kPa}$ and a temperature sensitivity of $17.1 \mathrm{mV} \mathrm{K}^{-1}$. The unique feature of pressure and temperature dual-parameter sensors is self-powering generated by thermoelectric effect.

\section{Integrated bimodal sensors}

Another approach is to integrate two individual sensors into one single pixel, and each of them responds to one specific stimuli. Theoretically, there can be many bimodal sensor combinations if the number of types of pressure sensors multiplies by that of temperature sensors. Four major classes of bimodal sensors that transduce bimodal stimuli are discussed in this section: (1) piezoresistive/thermoresistive; (2) capacitive/thermoresistive; (3) piezoresistive/thermoelectric; and (4) piezoelectric/thermoelectric pressure and temperature bimodal sensors. 


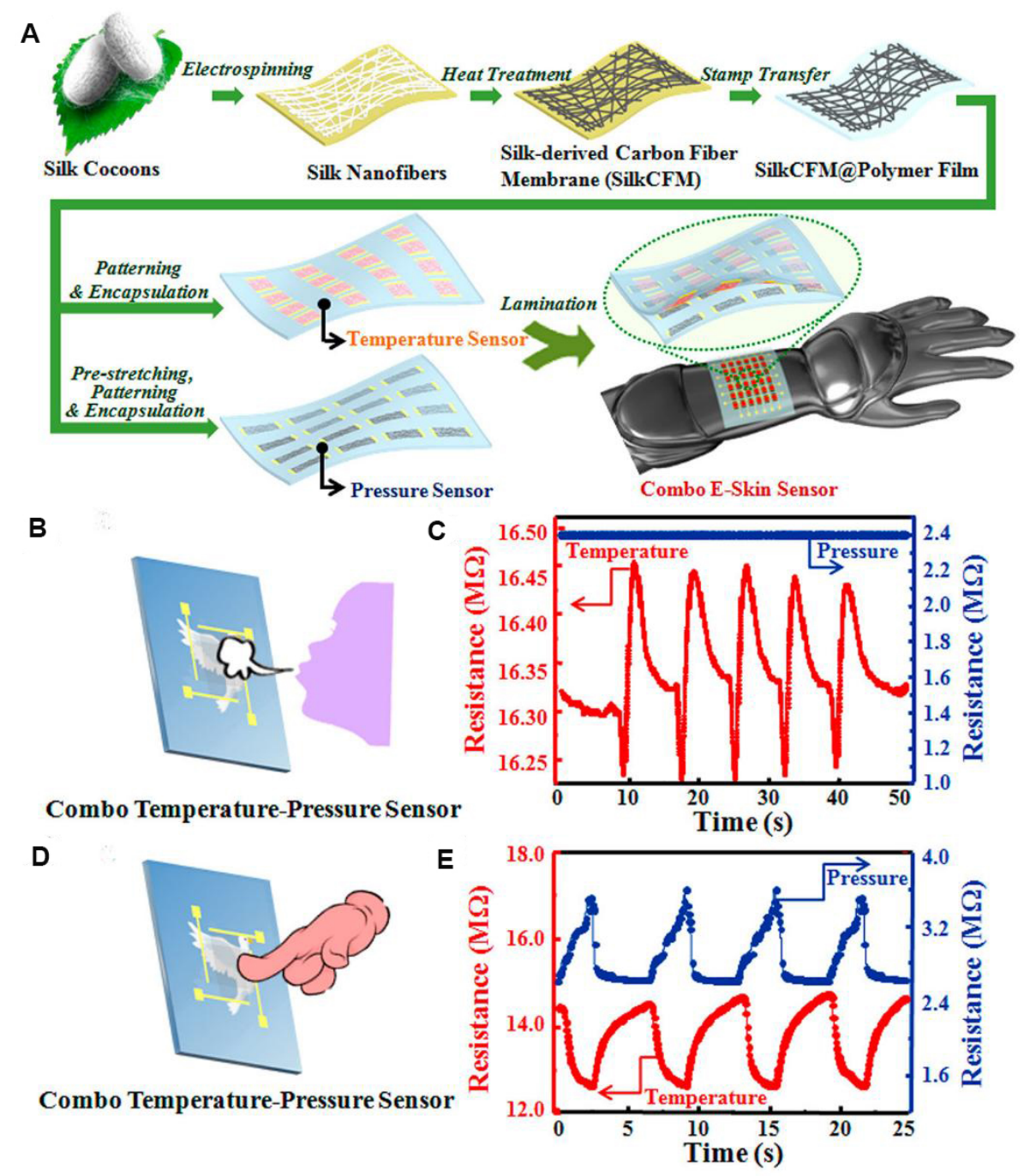

Figure 4. (A) Schematic illustration of the fabrication process of the combo temperature-pressure E-skin; and (B-E) schematic illustration and simultaneous sensing performance of the combo E-skin sensor under various stimuli. This figure is quoted with permission from Wang et al. ${ }^{[30]}$.

\section{Piezoresistive/thermoresistive-type integrated bimodal sensors}

The main difference between dual-parameter sensors and integrated bimodal sensors using piezoresistive/thermoresistive as active materials is the number of active materials or sensors. Only one active material is used for the former and two active materials are used for the latter. Gui et al ${ }^{[36]}$ reported a skin-like tactile sensor for simultaneous detection of pressure and temperature without signal interference. The pressure sensor was fabricated using wrinkled Au films as thermoresistive materials. The temperature sensor was composed of a coiled thread treated with thermoresistive ionic liquid. Jung et al ${ }^{\left[{ }^{[3]}\right]}$ demonstrated a transparent and flexible pressure and temperature bimodal sensor using ITO as an active material, which was embedded in a PDMS substrate, as observed in Figure 6. The thermoresistive pressure sensor was prepared by detaching the embedded ITO from a pyramid structured silicon mold, which had a broad sensing range from $100 \mathrm{~Pa}$ to $10 \mathrm{kPa}$ with a high stability of $10^{5}$ cycles. Classification of different surface textures was achieved through machine learning. The temperature sensor was prepared by ITO as thermal sensitive material with a zigzag shape using photolithography technique and had a detection limit of $-9{ }^{\circ} \mathrm{C}$. In addition, a $5 \times 5$ pixel pressure and temperature bimodal sensor was also demonstrated without 

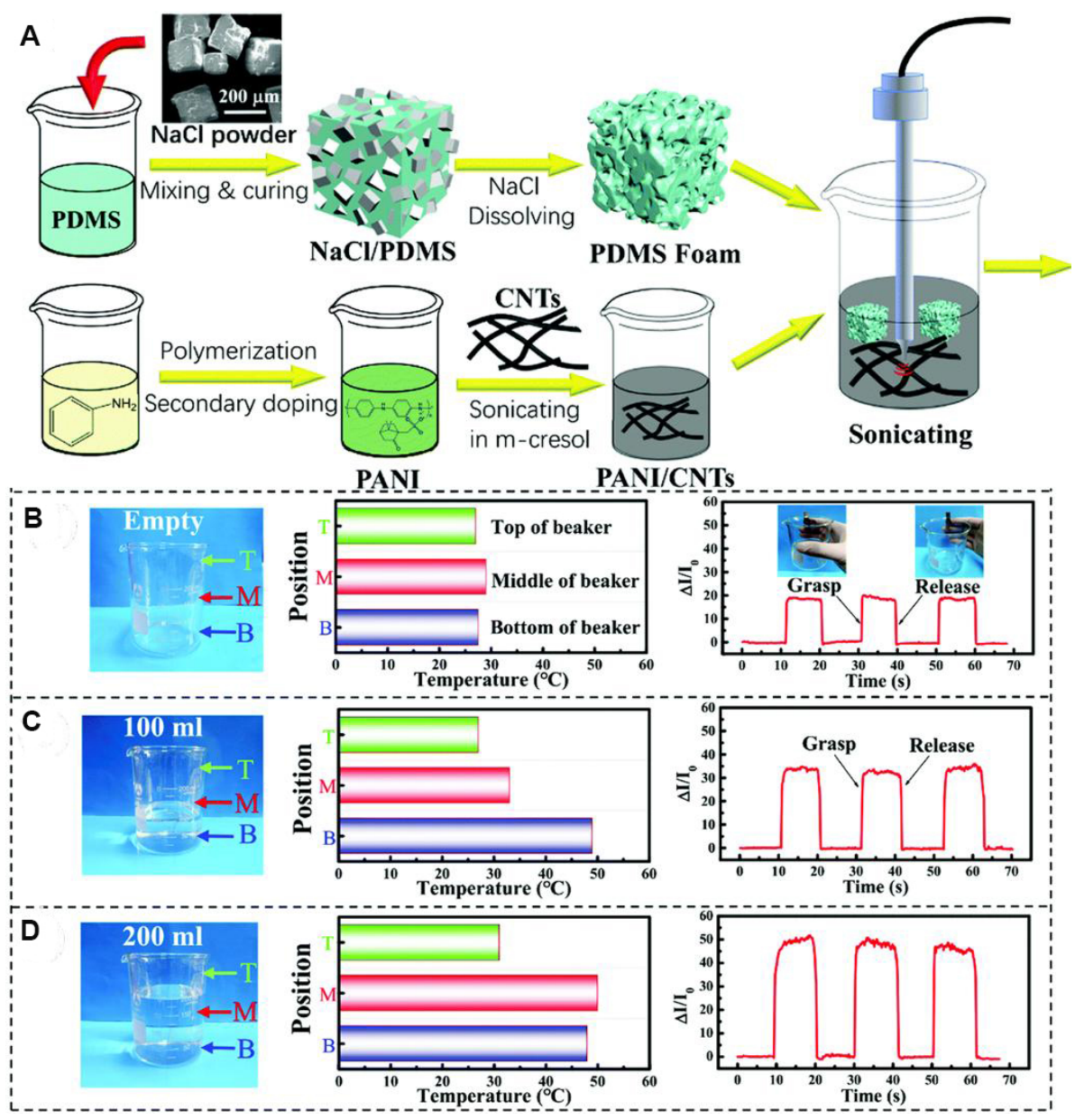

Figure 5. (A) Preparation process of PANI/CNTs dual-parameter sensors; and (B-D) application of PANI/CNTs dual-parameter sensors for temperature and pressure sensing. This figure is quoted with permission from Wang et al. ${ }^{[35]}$.

noticeable interference. Wang et al ${ }^{[38]}$ developed a pressure and temperature bimodal sensor integrating AgNP/SBS and PEDOT:PSS/SBS composites, which were used as piezoresistive and thermoresistive materials for pressure and temperature sensing, respectively. The pressure sensor shows an ultrahigh pressure sensitivity of $1185.8 \mathrm{kPa}^{-1}$ with an extremely low pressure detection limit of $2.4 \mathrm{~Pa}$, and stability of up to 5000 cyclic loading tests. A $4 \times 5$ pixel pressure and temperature bimodal sensor was further evaluated showing accurate detection of pressure and temperature signals without significant interference.

\section{Capacitive/thermoresistive-type integrated bimodal sensors}

Bae et al.$^{[39]}$ reported a bimodal tactile sensor that can simultaneously distinguish pressure and temperature signals by introducing a temperature independent material. By using the capacitive pressure transduction mechanism, the pressure sensor shows a sensitivity of $0.7 \mathrm{kPa}^{-1}$ to $25 \mathrm{kPa}$ with high linearity and fast response time within $50 \mathrm{~ms}$. By introducing a microstructured RGO as the thermoresistive material, the temperature sensor exhibits a temperature coefficient of resistance of $0.83 \% \mathrm{~K}^{-1}$ in the range of $22-70{ }^{\circ} \mathrm{C}$ with a response time of less than $100 \mathrm{~ms}$. Wu et al. ${ }^{[40]}$ presented a temperature and pressure bimodal textile sensors based on silkworm fibers [Figure 7]. The capacitive pressure sensor was prepared using Ag nanowires as electrodes and Ecoflex as dielectric insulating layer, showing a pressure sensitivity of 0.136 $\mathrm{kPa}^{-1}$, a response time of $0.25 \mathrm{~s}$, and stability of 5000 cycles. The thermoresistive temperature sensor employed a mixture of CNTs and ionic liquid as temperature sensitive materials to enhance the temperature 


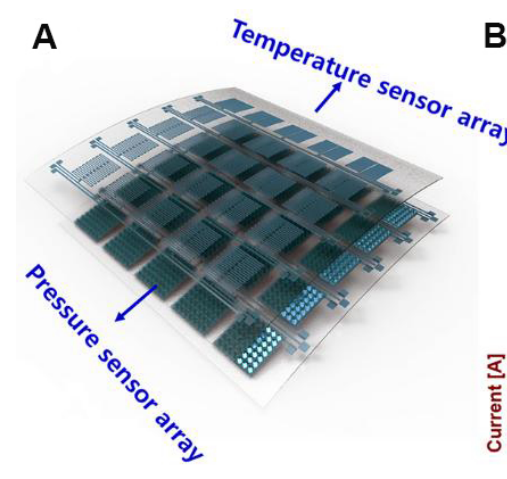

D

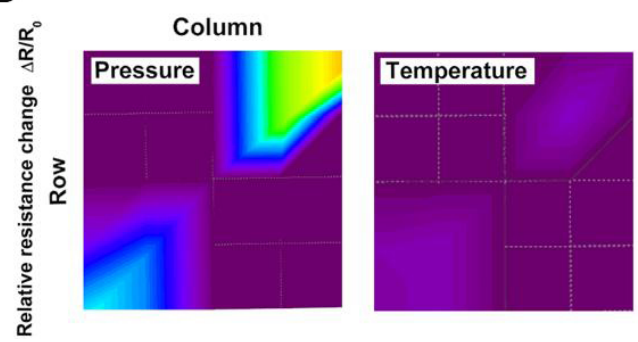

B
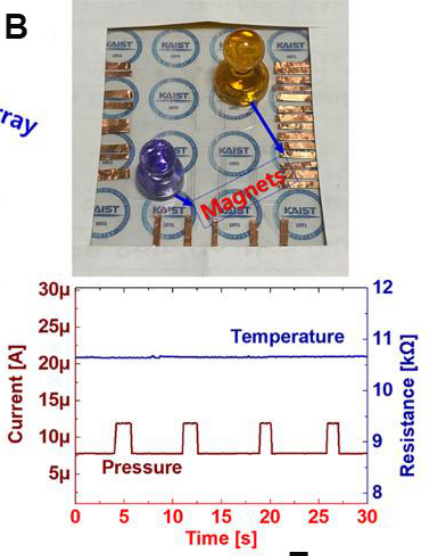

E

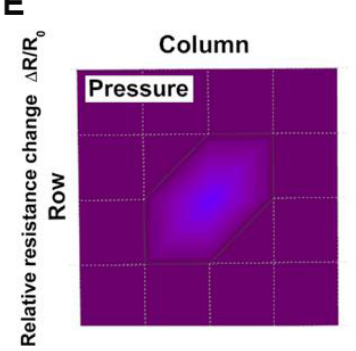

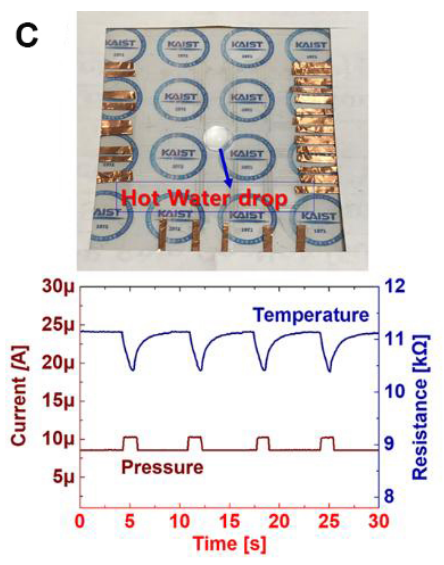

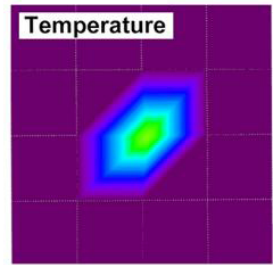

Figure 6. (A) Schematic view of bimodal sensor. Signal response and real-time mapping for (B, D) small magnets and ( , E) hot water droplet on bimodal sensor array. This figure is quoted with permission from Jung et al. ${ }^{[37]}$.

sensing performance, which displayed a temperature sensitivity of $1.23 \% / \mathrm{K}$. The combined pressure and temperature sensor arrays were assembled by weaving technique, with the underlying layer for pressure detection and top layer for temperature detection. Shin et al. ${ }^{[4]}$ realized a self-healing and conductivity soft temperature and pressure bimodal sensor by an interpenetrating polymer network (IPN) system, which was based on chemical/ionic cross linked $\mathrm{Fe}^{3+} / \mathrm{PAA}$ and physically cross-linked PVA. The ionic conductivity of IPN hydrogels enables the ability to sense pressure and temperature signals. The obtained bimodal tactile sensor can simultaneously detect folding and pressure motions, and heat signals at maximum of $90{ }^{\circ} \mathrm{C}$.

\section{Piezoresistive/thermoelectric-type integrated bimodal sensors}

The type of pressure and temperature bimodal sensor was mostly reported by Jung et al..$^{[42,43]}$ and Kim et al. ${ }^{[44]}$. Jung et al.$^{[42]}$ presented a pressure and temperature bimodal sensor using paper as substrate that was prepared by inkjet printing [Figure 8]. The pressure sensor was prepared using microstructured pyramid PDMS with MWCNT conducting ink and shows a sensing range from $100 \mathrm{~Pa}$ to $5 \mathrm{kPa}$. The thermoelectric temperature sensor was based on a thermocouple composed of conductive PEDOT:PSS and AgNPs inks and shows a wide sensing range over $150{ }^{\circ} \mathrm{C}$. Furthermore, a $5 \times 5$ pixel bimodal sensor demonstrated negligible signal interference and fast response for pressure and temperature stimuli. Jung et al.$^{[43]}$ in another work demonstrated an integrated nanocellulose-based pressure and temperature bimodal sensor. The pressure sensor based on piezoresistive transduction principle was prepared by inkjet printing conducting electrode on the TEMPO-oxidized-nanocellulose and placing the counter electrode on the nanocellulose. The as-prepared pressure sensor exhibits a high sensitivity in a working range from $500 \mathrm{~Pa}$ to $3 \mathrm{kPa}$. The temperature sensor combined the conductive materials PEDOT:PSS, AgNPs, and CNTs to form a thermocouple on the nanocellulose. The thermoelectric temperature sensors can produce a voltage of 1.7 $\mathrm{mV}$ with $125 \mathrm{~K}$ temperature gradient. 


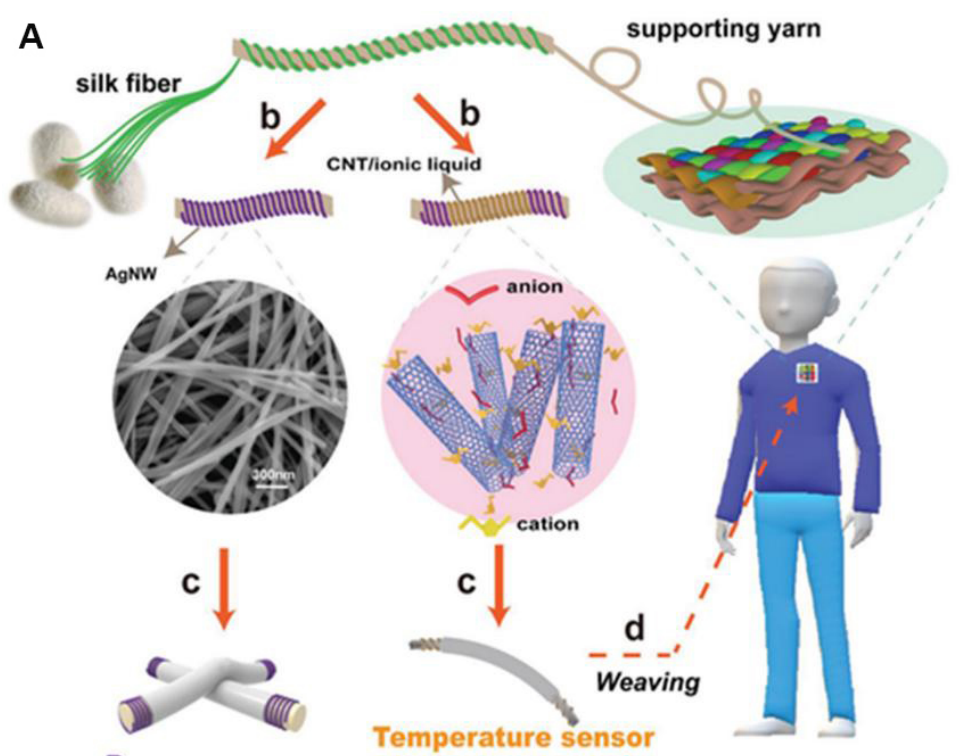

B

\section{Pressure sensor}

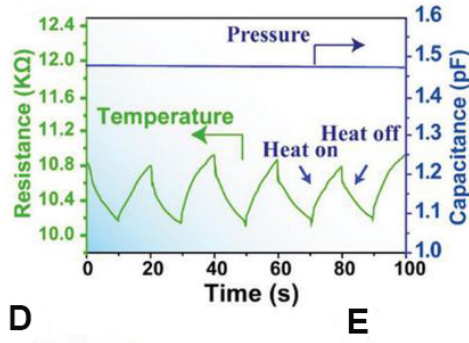

Bottle with 2D Temperature hot water Sensing textile

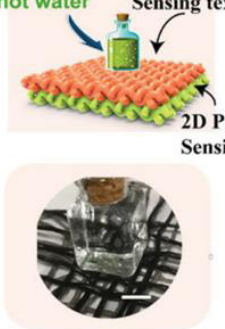

Combo E-textile sensors

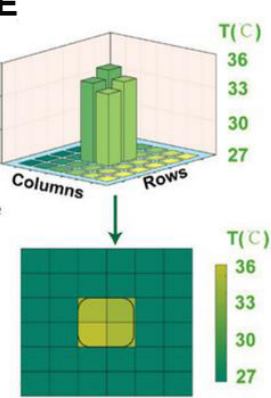

Temperature
C

Combo E-textile sensors

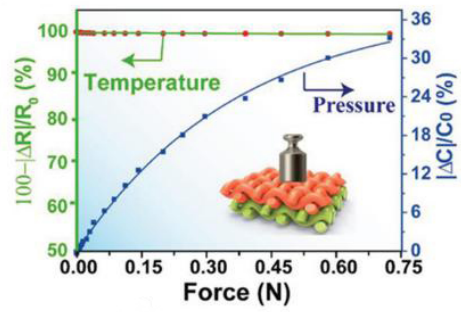

$\mathbf{F}$

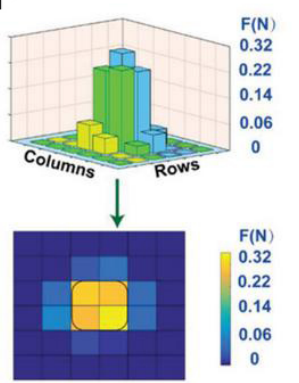

Pressure

Figure 7. (A) Scheme of fabrication of silk fibers-wrapped combo temperature-pressure sensors; and (B-F) pressure and temperature performance of the combo textile temperature-pressure sensors. This figure is quoted with permission from Wu et al. ${ }^{[40]}$.

\section{Piezoelectric/thermoelectric-type integrated bimodal sensors}

This type of pressure and temperature bimodal sensor was reported by Zhu et al. ${ }^{[45,46]}$. We developed a flexible pressure and temperature bimodal sensor using organic piezoelectric PVDF and thermoelectric PANI as sensitive materials constructed in a sandwiched structure, as displayed in Figure $9^{[45]}$. The pressure function was evaluated by human motion, such as elbow bending, pronunciation, and artery pulse. The integration of thermoelectric PANI enabled the device to sense temperature with a sensitivity of $45.5 \mathrm{mVK}^{-1}$ and response time of $1.2 \mathrm{~s}$. Recently, our group also demonstrated a 3D processing technique combined with laser engraving technique and screen-printing method to integrate pressure and temperature bimodal sensors using piezoelectric $\mathrm{P}(\mathrm{VDF}-\mathrm{TrFE})$ and thermoelectric PANI as active materials, as shown in Figure $10^{[46]}$. The pressure sensor shows superior pressure sensitivity in a broad sensing range from $100 \mathrm{~Pa}$ to $20 \mathrm{kPa}$. The temperature sensor presents high temperature sensitivity of $109.4 \mu \mathrm{VK}^{-1}$ with rapid response 


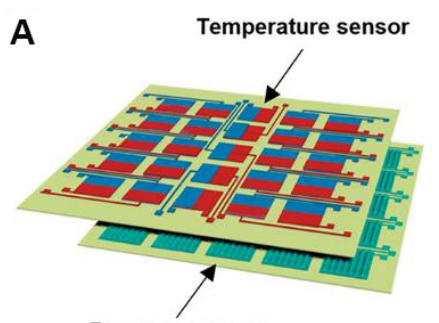

Pressure sensor

C

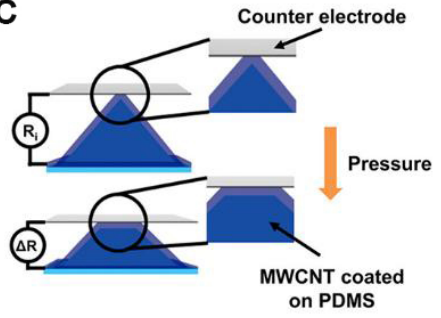

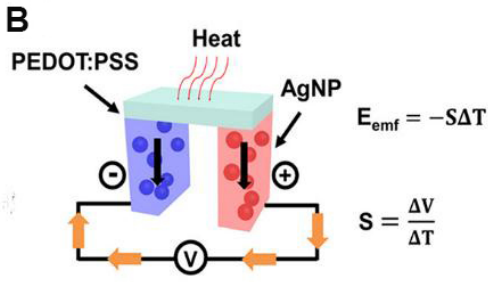

D

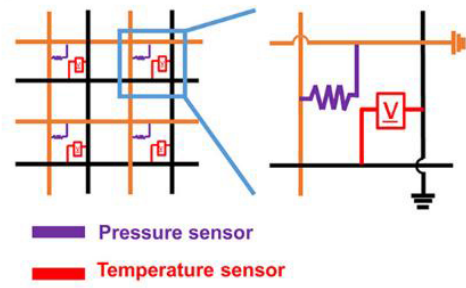

Figure 8. (A) Paper based flexible pressure and temperature bimodal sensor; (B) principle of the thermoelectric temperature sensor; (C) capacitive pressure sensing mechanism; and (D) equivalent circuit of the bimodal sensor matrix in a single pixel. This figure is quoted with permission from Jung et al. ${ }^{[42]}$.

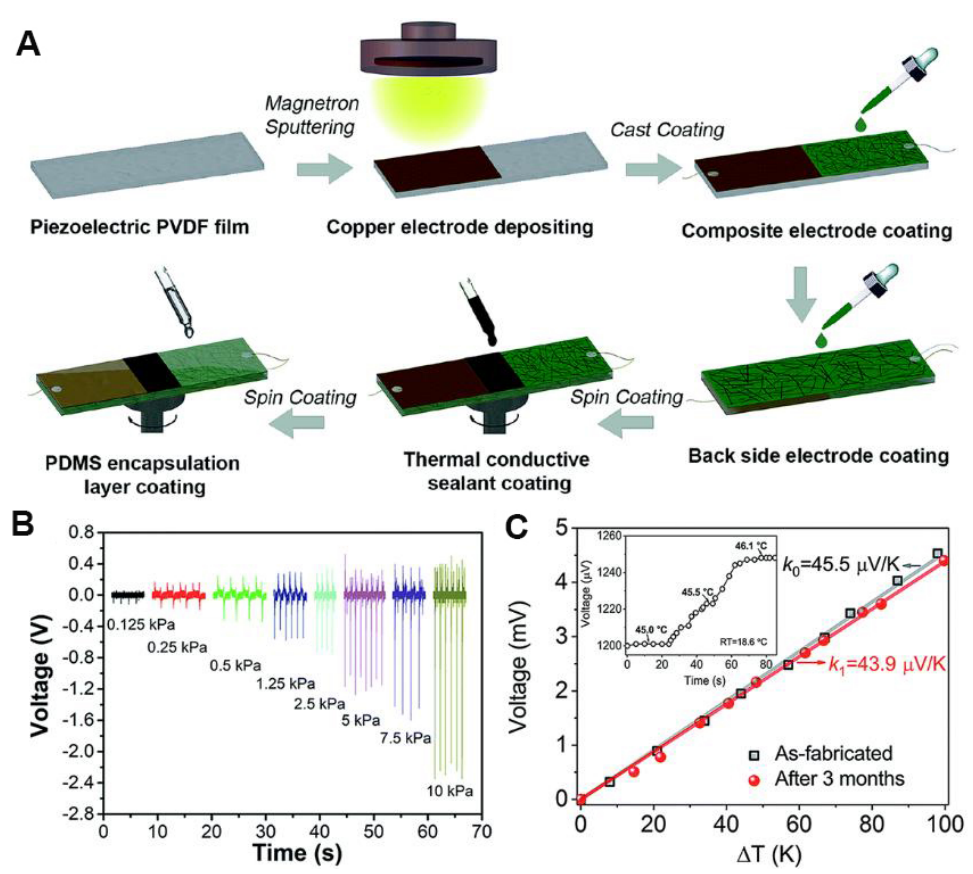

Figure 9. (A) Fabrication process of a temperature sensitive piezoelectric device; and (B) pressure sensitivity and (C) linear temperature response of the bimodal sensor after three months with the inset showing temperature sensitivity. This figure is quoted with permission from Zhu et al. ${ }^{[45]}$.

time of $0.37 \mathrm{~s}$. Furthermore, a $4 \times 4$ pixel bimodal sensor was integrated, presenting negligible signal interference and functioned battery free due to the self-powering working principles of piezoelectric and thermoelectric materials.

Other integrated bimodal sensors

In addition, other bimodal sensors such as pyro-piezoelectric sensors have also been employed to construct 


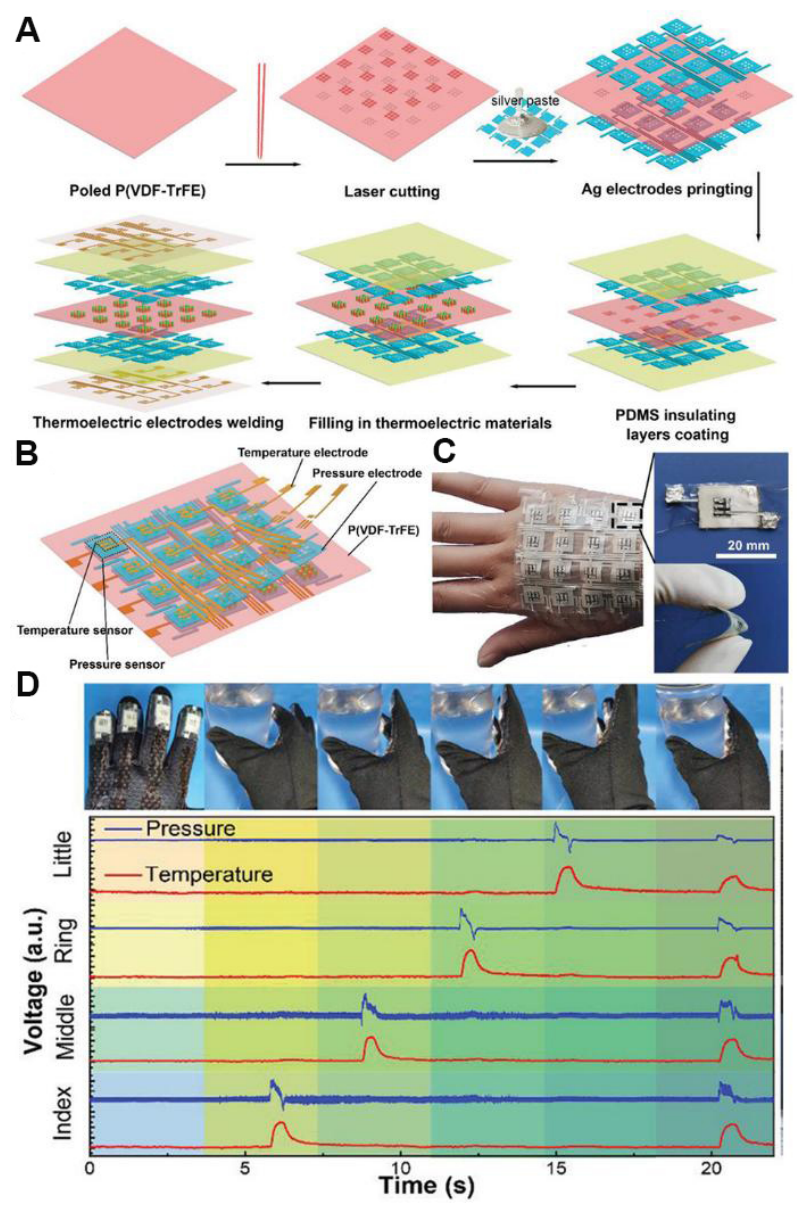

Figure 10. (A) Fabrication process of the bimodal sensor array; (B) illustration of the $4 \times 4$ pixel bimodal sensor array; (C) photographs of sensor array attached on hand; and (D) an application of the sensors attached to a glove in response to finger motions while holding a warm cup. This figure is quoted with permission from Zhu et al. ${ }^{[46]}$.

pressure and temperature bimodal sensor. For example, Song et al. ${ }^{[4]}$ demonstrated a ferroelectric $\mathrm{BaTiO}_{3}{ }^{-}$ based pyro-piezoelectric bimodal sensor to simultaneously detect temperature and pressure signals. The pressure sensitivity and temperature sensitivity were found to be about $0.044 \mathrm{VkPa}^{-1}$ and $0.048 \mathrm{~V}^{\circ} \mathrm{C}^{-1}$, respectively. In addition, a $4 \times 4$ pixel bimodal sensor was formed to detect continuous temperature and pressure changes caused by finger motion.

\section{INTEGRATION OF TACTILE SENSORS: POWER SUPPLY, WIRELESS COMMUNICATION AND SIGNAL PROCESS CIRCUIT}

Apart from the principles and classification mentioned above, it is also of great importance for the pressure and temperature tactile sensors to be integrated with other electric components such as power supply, wireless communication, and signal process on readout circuits, which will ensure accurate and real-time signal processing and data transmission. It is highly desired and even required for flexible tactile sensors to be self-powered without the need of batteries. To ensure continuous signal monitoring and data transmission, research on integration of sensors with signal process circuit and wireless communication has also been widely performed. In this section, the integration of tactile sensors from the aspects of power supply, wireless communication, and signal process circuits is addressed. 


\section{Power supply: energy harvesting and energy storage techniques}

Traditional tactile sensors are usually powered by bulky lithium batteries which have to be recharged frequently. However, it severely limits the development of next generation tactile sensors, because of the urgent requirement of lightweight, flexibility, and autonomous and continuous operation. This section focuses on energy harvesting techniques directly extracting energy from the ambient environment and converting it into electricity, together with energy storage techniques to be introduced. The benefits and shortcomings of energy harvesting and storage techniques are summarized in Table 3.

Energy harvesting techniques: photovoltaics, thermoelectrics, piezoelectrics, and triboelectrics

Energy harvesting techniques such as photovoltaics, thermoelectrics, piezoelectrics and triboelectrics can allow the utilization of tactile sensors without bulky batteries or supercapacitors. This subsection highlights the recent advances in energy harvesting techniques.

\section{Photovoltaics}

Photovoltaics can direct convert light into electricity using photoelectric materials, which are able to release electrons when illuminated with solar light. When these photons' energy is greater than the work function of the material, electrons are emitted, and a current is then generated. The disadvantage of photovoltaics is that solar energy is neither consistent nor predictable, which restricts practical application in tactile sensors. However, the photovoltaics approach can be used together with other energy harvesting and storage techniques in a cost-effective and environmentally friendly way. Photovoltaics can be categorized into four types according to the photovoltaic materials: (1) organic photovoltaics; (2) dye-sensitized photovoltaics; (3) inorganic photovoltaics; and (4) lead halide perovskite and quantum dots.

\section{Organic photovoltaics}

Organic photovoltaics have the advantages of lightweight and flexible. However, low energy conversion efficiencies and poor environmental stability limit their utilization in tactile sensors. The advances in the development of new semiconducting polymers and absorbers have enabled power conversion efficiencies exceeding $10 \%{ }^{[48]}$. Dye-sensitized solar cells, also called Grätzel cells ${ }^{[49]}$, are based on mesoscopic photoelectrode $\mathrm{TiO}_{2}$ materials applied with metal free dye sensitizer for photon absorbing. Recently, Michaels et al.$^{[50]}$ developed a highly efficient organic dye-sensitized solar cell based on copper (II/I) electrolyte with remarkable conversion efficiency up to $34 \%$ by a co-sensitization strategy. It was achieved by tailoring the cells to be suitable for ambient light harvesting indoors, and it could power machine learning for IoT application. Inorganic photovoltaics such as commercial polycrystalline and monocrystalline Si have dominated $90 \%$ of the market, mainly applied outdoors for many years owing to the high conversion efficiency. However, narrower bandgap and higher processing costs limit inorganic photovoltaics for indoor light-harvesting applications. Recently, halide perovskite materials have drawn a lot of research interest due to outstanding performance of high efficiency and low fabrication cost compared with other traditional photovoltaic approaches. Quantum dots used as absorbing photovoltaic materials are another promising candidate for next generation photovoltaics thanks to their tunable band gap.

\section{Thermoelectrics}

A thermoelectric generator (TEG) can directly convert waste heat energy into useful electricity by taking advantage of the temperature difference. The performance of TEG is determined by the figure of merit (ZT) calculated as follows: $Z T=S^{2} \sigma T / \kappa$, where $S$ stands for the Seebeck coefficient, $\sigma$ is the electrical conductivity, $\kappa$ represents for the thermal conductivity, and $T$ means the absolute temperature. Numerous efforts were devoted to developing TEG by Wang et al. ${ }^{[51,52]}$, Feng et al. ${ }^{[53]}$, and Sheng et al. ${ }^{[51-54]}$. In a typical work, we developed a flexible pressure sensor which was self-powered by a thin-film $\mathrm{TEG}^{[51]}$, as shown in Figure 11 . 
Table 3. Comparison of advantages and disadvantages of energy harvesting and storage techniques

\begin{tabular}{llll}
\hline Energy type & Technology & Advantages & Disadvantages \\
\hline Energy harvesting & Photovoltaics & High conversion efficiencies, flexible & High processing cost \\
& Thermoelectrics & Reliable source of energy, Heating and cooling in one system & Low efficiency \\
& Piezoelectrics & Low operation frequency, high energy density, flexible & Difficult integration \\
& Triboelectrics & High energy density, low cost, flexible & Difficult integration \\
Energy storage & Supercapacitor & High lifetime, high power density & Low energy density, low working voltage \\
& Battery & High energy density, high working voltage & Low power density, low lifetime \\
\hline
\end{tabular}

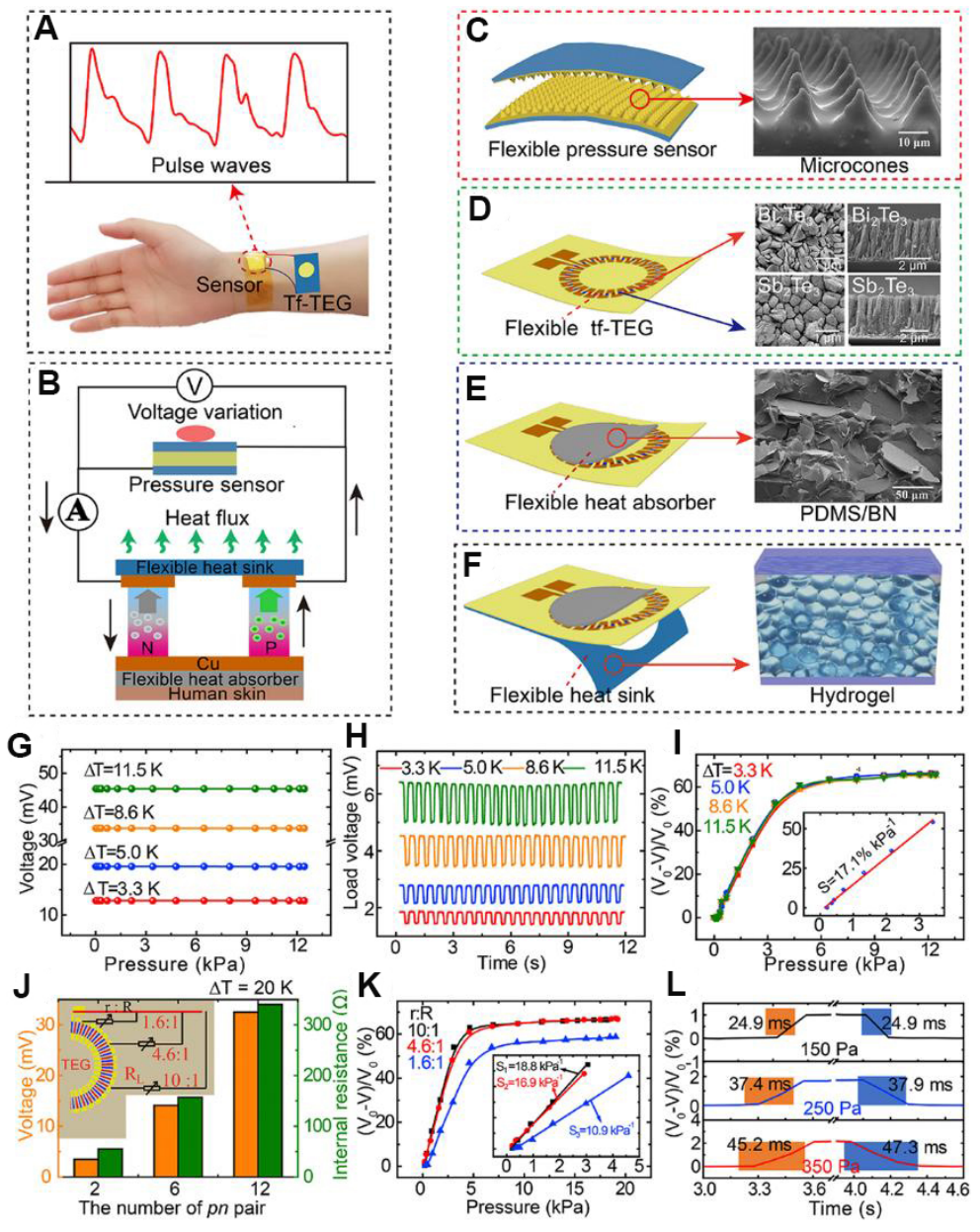

Figure 11. (A-F) Schematic descriptions and morphology of the self-powered pressure sensing system integrating tf-TEG; and (G-L) performance evaluation of the self-powered pressure sensing system. This figure is quoted with permission from Wang et al. ${ }^{[51]}$.

The obtained thin film TEG can generate an output voltage of $78 \mathrm{mV}$ with a temperature difference of $20 \mathrm{~K}$. For the first time, the flexible pressure sensor self-powered by thermoelectric generator without external power supply ensured consistent monitoring of various physiological signals. In addition, Wang et al ${ }^{[52]}$ also prepared high performance $\mathrm{Bi}_{2} \mathrm{Te}_{3}$ films by the screen-printing method. The promoted carrier migration improved the electrical conductivity by around seven times, achieving a maximum power factor of 4.65 $\mu \mathrm{Wcm}^{-1} \mathrm{~K}^{-2}$. A TEG device with a single leg was prepared, presenting a maximum output voltage of 11.34 $\mathrm{mV}$ with a temperature difference of $105 \mathrm{~K}$. 


\section{Piezoelectrics and triboelectrics}

Mechanical energy harvesting such as piezoelectric and triboelectric energy harvesting can capture energies from surroundings such as human activities, wind, and waves. Park et al ${ }^{[55]}$ proposed a pulse sensor selfpowered by piezoelectric PZT. The result shows that the pulse sensor had a sensitivity of about $0.018 \mathrm{kPa}^{-1}$ and response time of around $60 \mathrm{~ms}$. Chen et al. ${ }^{[5]}$ demonstrated a self-powered piezoelectric sensor based on $[\mathrm{P}(\mathrm{VDF}-\mathrm{TrFE})] / \mathrm{BaTiO}_{3}$ micropillar arrays, which were prepared by nanoimprinting technology for energy harvesting. The as-prepared nanogenerator produced an output voltage of $13.2 \mathrm{~V}$ and a current density of $0.33 \mu \mathrm{Acm}^{-2}$, and that value was enhanced by 7.3 times compared with bare P(VDF-TrFE) film. In a typical work by Fan et al. ${ }^{[57]}$, they demonstrated a highly efficient pressure sensor self-powered by flexible triboelectric nanogenerator. The power generation was enhanced by the introduction of microstructured arrays with a pyramid-like microstructure device, producing a maximum voltage of $18 \mathrm{~V}$ at a current density of around $0.13 \mu \mathrm{A} / \mathrm{cm}^{2}$. Furthermore, the as-prepared self-powering pressure sensor was capable of sensing a tiny water droplet and falling feather with a detection limit of $\sim 13 \mathrm{mPa}$.

\section{Energy storage techniques: batteries and supercapacitors}

Energy storage techniques such as batteries and supercapacitors are the major energy storage sources in tactile sensors. The recent advances in energy storage techniques are given in this subsection.

\section{Batteries}

Batteries are widely used in our daily life. Typically, batteries can be mainly classified into two categories: primary and secondary batteries. Primary batteries are disposable batteries while secondary batteries are rechargeable ones. Alkaline batteries, silver-oxide batteries, mercury batteries, and zinc carbon batteries belong to primary batteries, while lead-acid batteries and lithium batteries are the secondary batteries. Among these, lithium batteries have revolutionized our daily lives and are widely used in consumer electronic devices thanks to their light weight and high energy density. Considerable attention has been paid to the development of flexible lithium batteries. Typically, lithium batteries are composed of an anode, a cathode, an electrolyte, and a separator ${ }^{[58]}$. The cathode is comprised of materials such as $\mathrm{LiCoO}_{2}, \mathrm{LiMn}_{2} \mathrm{O}_{4}$, or $\mathrm{LiFePO}_{4}$. The anode is composed of materials such as carbon nanofiber, carbon nanotubes, or graphene. The electrolyte is a mixture of an electrolyte salt such as $\mathrm{LiPF}_{6}$ or $\mathrm{LiBF}_{4}$ in organic solvents. With the rapid need for a power supply for tactile sensors, fiber lithium batteries have become one of the attractive flexible power sources in recent years. For example, Pan et al. ${ }^{[59]}$ developed aligned N-CNT films prepared by CVD method working as flexible anodes to fabricate lithium batteries showing electrical conductivity of 410 $\mathrm{Scm}^{-1}$. Additionally, the as-prepared lithium batteries exhibited a high capacity of $390 \mathrm{mAhg}^{-1}$.

\section{Supercapacitors}

Supercapacitors are also excellent electronic devices for energy storage for tactile sensors. Supercapacitors can be classified into two types according to the electric storage mechanisms: double-layer capacitance mechanism and pseudocapacitive mechanism. Double-layer capacitance is electrostatic and pseudocapacitive is electrochemical. Wang et al. ${ }^{[60]}$ developed a flexible pressure and temperature bimodal sensor based on a piezoelectrically active supercapacitor. The supercapacitor was composed of paper immersed in PEDOT:PSS as electrodes, PVDF as separator, and a mixture of $[\mathrm{BMIM}]\left[\mathrm{BF}_{4}\right] / \mathrm{ETPTA}$ as electrolyte. By coupling PVDF separator into the supercapacitor, the as-prepared piezoelectrically active supercapacitor could work as a self-powering pressure and temperature bimodal tactile sensor. It was confirmed that the supercapacitor was able to charge up to $150 \mathrm{mV}$ in $300 \mathrm{~s}$ by tapping fingers. Wang et al ${ }^{[61]}$ reported stretchable supercapacitor-based E-skin for continuous monitoring of human activities. The supercapacitors were fabricated by using AgNW/MNW as electrodes and PVA-KOH as solid electrolytes. The as-prepared stretchable supercapacitors showed a large capacitance of $371 \mathrm{mFcm}^{-2}$ when the current density was $1 \mathrm{mAcm}^{-2}$. 


\section{Wireless communication: NFC, BLE, ZigBee, and Wi-Fi}

Wireless communication is highly desirable in smart tactile sensors. There are mainly four wireless communication protocols available for data communication with third-party computing devices: NFC, BLE, ZigBee, and Wi-Fi. The characteristics of wireless communication protocol are summarized in Table 4.

NFC

Near field communication (NFC) is a short-range wireless technology that makes two devices communicate with each other. NFC transmits data through electromagnetic radio fields. Han et al. ${ }^{[62]}$ used NFC power delivery and wireless communication for temperature and pressure tactile sensor, as shown in Figure 12. The key features of these systems were the experimental and theoretical investigations of sensor operation and NFC wireless modes.

\section{$B L E$}

Bluetooth low energy (BLE) is based on Bluetooth 4.0 standard. It works at $2.4 \mathrm{GHz}$, almost in opposition to NFC technology, with the difference that BLE has higher reading distances of up to $100 \mathrm{~m}$. For example, Song et al. ${ }^{[63]}$ proposed a wireless sweat sensor patch applied for continuous monitoring sweat bioinformation such as $\mathrm{pH}$ and $\mathrm{Na}^{+}$, as shown in Figure 13. The sweat sensor patch was powered by triboelectric nanogenerators, and sensor data were wirelessly transmitted to mobiles through BLE on flexible circuit.

\section{ZigBee}

ZigBee is a wireless technology operated on an IEEE 802.15.4-based protocol developed by the member companies of the ZigBee Alliance. It is a cost-effective and low-energy wireless network extensively adopted for monitoring applications where the reading distance is in the range of 10-100 $\mathrm{m}$.

\section{Wi-Fi}

Wi-Fi is a wireless networking technology that allows devices to transmit data without cables. It is operated through wireless protocol based on 802.11 IEEE network standard. It has been widely used in devices such as computers, mobiles, and other equipment to interface with the Internet.

\section{Signal process circuits}

In the traditional tactile sensor array measurement, many connector wires are demanded for acquiring all sensing unit data in $\mathrm{M}$ column $\times \mathrm{N}$ row arrays. Scanning approaches of sandwich structured tactile sensor arrays with reduced wires $(\mathrm{M}+\mathrm{N})$ have been proposed by only taking potential measurements in shared wires. However, the crosstalk effect will still occur due to parasitic parallel paths, which will influence the electric measurements of signal readouts. Signal isolation circuits are therefore urgently needed to overcome the crosstalk effects between the sensor units. In this section, some typical studies on signal process circuits for tactile sensors to reduce the crosstalk effect are introduced. Luo et al. ${ }^{[64]}$ designed a modified electrical grounding circuit to eliminate crosstalk and parasitic effects of $32 \times 32$ piezoresistive tactile textures matrix, as shown in Figure 14A. The voltage difference across all lines was maintained at the ideal $0 \mathrm{~V}$, except the measuring one controlled by a 32 SPDT switch. A 32:1 analog switch was employed as a multiplexer to scan the circuit through one column by one. All the electronic components were performed by a microcontroller, and every signal was then transmitted serially to a computer. Chen et al. ${ }^{[13]}$ proposed a piezoelectric sensor array integrating a portable signal processing circuit, as shown in Figure 14B. The potential outputs of all sensing units in $12 \times 12$ pixels were first periodically selected by the circuit, and after that signals were sent to the processing module. An offset circuit was applied to ensure that all the signals acquired were positive to meet the requirement. Finally, output signals were further processed through a notch filter to reduce electrical noise in the spectrum. An et al. ${ }^{[65]}$ developed a multiplexed capacitive bimodal tactile sensor array, 
Table 4. Summary of the characteristic of wireless communication protocol

\begin{tabular}{lllll}
\hline Protocol & Frequency/Hz & Data rate/bps & Distance/m & Power consumption/mA \\
\hline NFC & $13.56 \mathrm{M}$ & $424 \mathrm{k}$ & 0.2 & 0.2 \\
BLE & $2.4 \mathrm{G}$ & $2 \mathrm{M}$ & 100 & 4.8 \\
ZigBee & $2.4 \mathrm{G}$ & $20-250 \mathrm{k}$ & $10-100$ & 150 \\
Wi-Fi & $2.4 \mathrm{G} / 5.1 \mathrm{G}$ & $11 \mathrm{M}+$ & 80 & 210 \\
\hline
\end{tabular}
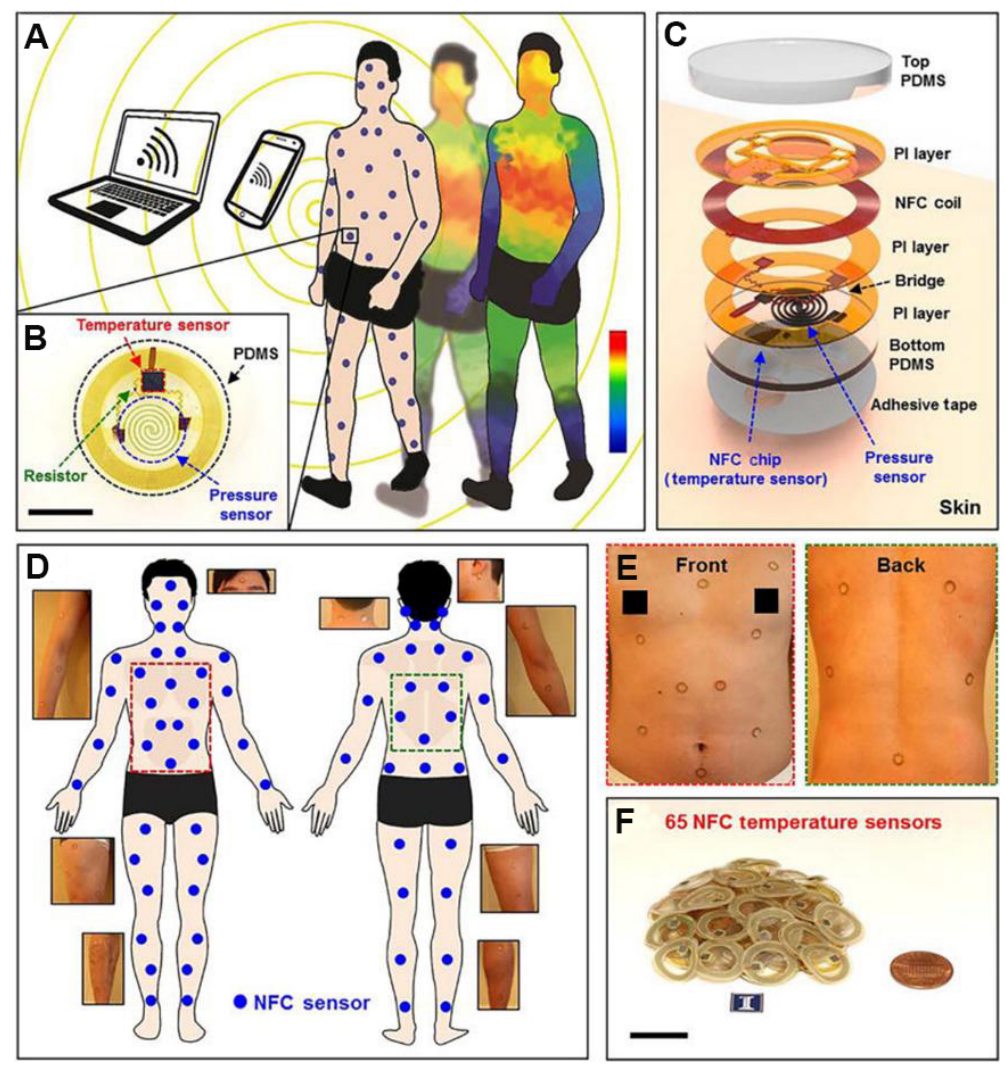

Figure 12. Concept illustrations, schematic diagrams and photographs of NFC wireless, battery-free epidermal sensors used for fullbody monitoring. This figure is quoted with permission from Han et al. ${ }^{[62]}$.

which was able to detect pressure and skin temperature simultaneously using mobiles. The circuit was composed of a screen, a transmitter, a driving unit, and a receiving unit, as shown in Figure $14 \mathrm{C}$. A differential circuit was employed for the receiver to detect low differences in capacitance. The multiplier and a low-pass filter can block noise signals. In this way, finger touch detection on the screen display can be achieved. In summary, a modified electrical grounding circuit is usually used for the piezoresistive sensor array to eliminate the crosstalk and parasitic effects. An offset and amplify circuit is adopted for piezoelectric sensor arrays to transduce the signals. For the capacitive sensor array, a differential circuit is required to detect low differences in capacitance.

\section{CONCLUSION AND OUTLOOK}

Recent advances in pressure and temperature tactile sensors are discussed in this review. A brief overview of pressure sensing mechanisms, such as piezoresistivity, capacitance, piezoelectricity, and triboelectricity, and temperature transduction mechanisms, such as thermosresistivity and thermoelectricity, are summarized. 
A

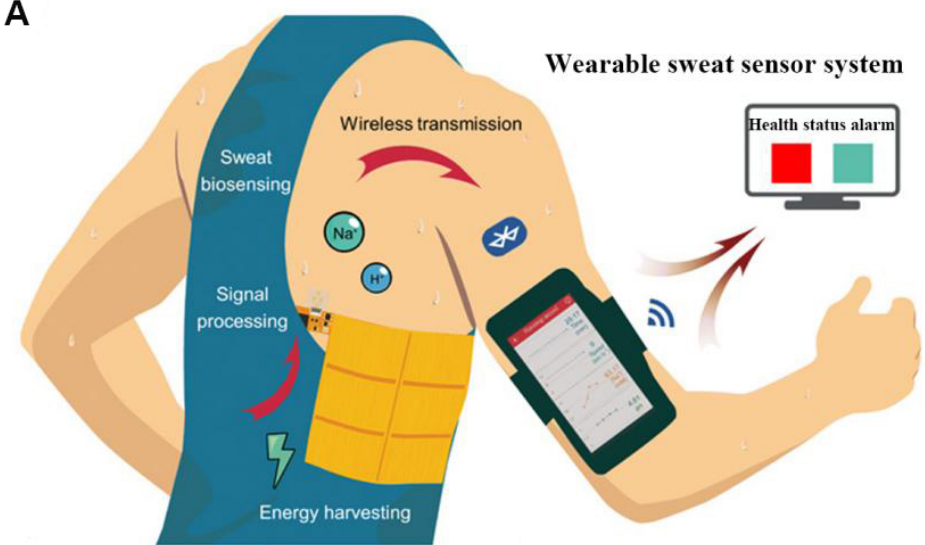

D

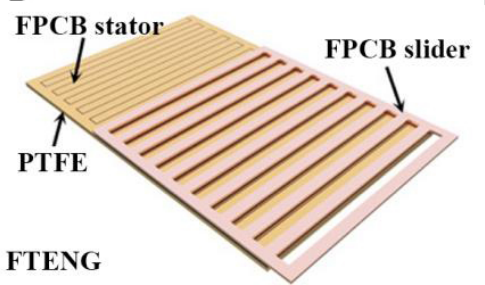

B

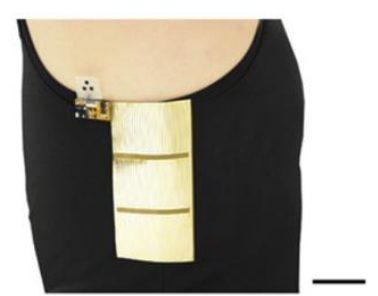

C

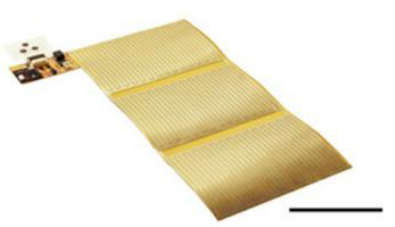

$\mathbf{F}$

E

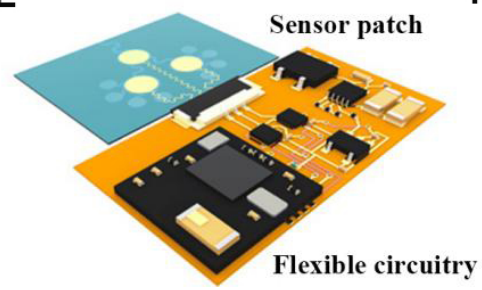

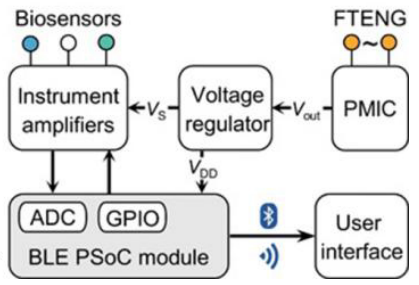

Figure 13. Battery-free $\mathrm{FWS}^{3}$ for BLE wireless and noninvasive molecular monitoring. This figure is quoted with permission from Song et al. ${ }^{[63]}$.

A

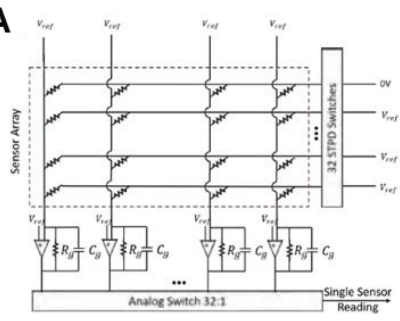

B

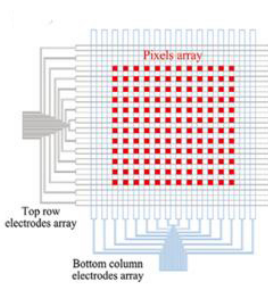

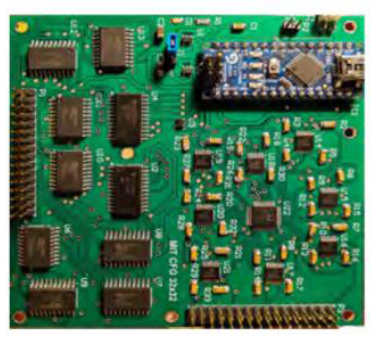

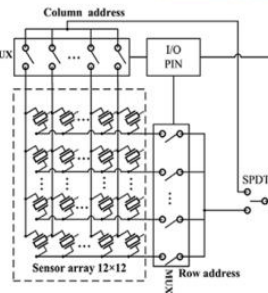

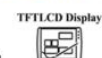

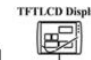
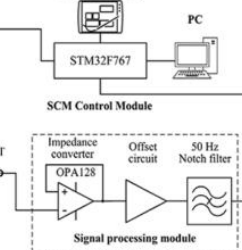

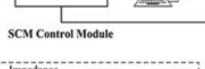

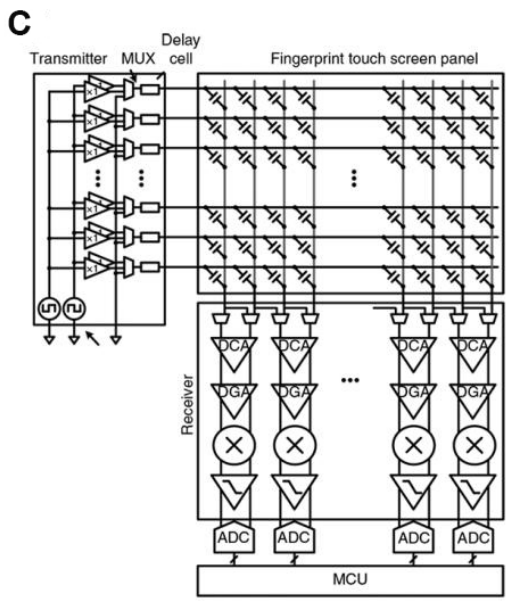

Figure 14. (A) A modified electrical grounding circuit and designed PCB board for $32 \times 32$ piezoresistive tactile arrays. This figure is quoted with permission from Luo et al. ${ }^{[64]}$. (B) Signal process circuit for the capacitive bimodal tactile sensor array. This figure is quoted with permission from Chen et al. ${ }^{[13]}$. (C) Designed readout circuit for fingerprint sensor. This figure is quoted with permission from An et al. ${ }^{[65]}$.

Among them, some critical parameters of pressure/temperature tactile sensors including sensitivity, sensing range, hysteresis, and response/recovery time are given in detail. According to the sensor units and transduction principles, bimodal temperature and pressure tactile sensors have been categorized into two types: dual-parameter sensors and integrated bimodal sensors. In addition to the principles and classification mentioned above, it is also of great importance for the pressure and temperature tactile sensors to be integrated with other electric components such as power supply, wireless communication, and 
signal process on readout circuits, which enable continuous signal monitoring. However, despite the significant progress in pressure/temperature tactile sensors, there are still many challenges to face. In the future, the following challenges and opportunities for the research of tactile sensors should be further considered:

(1) Scalable large area fabrication and high-level integration techniques need to be developed to ensure tactile sensors for the industrialization and common life applications. The fabrication of tactile sensors usually requires a complicated or patterning process. Conventional techniques such as photolithography, electron beam lithography, and focused ion-beam lithography methods are time-consuming as well as unsuitable for large-scale fabrication. Printed electronic circuits, e.g., using inject printing, screen printing, and $3 \mathrm{D}$ printing techniques, are highly desired due to the advantages of low cost and simplicity in largescale processability.

(2) Biocompatibility and biodegradability are also important functionalities of tactile sensors for practical applications. Eco-friendly tactile sensor systems developed from biocompatibility and biodegradability materials can reduce the amount of electronic waste, thus protecting the environment. In addition, the utilization of biocompatibility and biodegradability materials can avoid allergic reactions or foreign body reactions. Suitable materials such as naturally or synthetic degraded materials are desired to degrade sensor systems.

(3) Research on tactile sensors should be combined with recent progress in signal monitoring and wireless communication, ensuring accurate and real-time detection of motions and biological signals. Novel wireless communication techniques such as $5 \mathrm{G}$ or RFID are promising for wireless communication in flexible tactile sensors.

\section{DECLARATIONS}

\section{Authors' contributions}

Administrative, technical, and material support: Yu J, Zhang K, Deng Y

\section{Availability of data and materials}

Not applicable.

\section{Financial support and sponsorship}

This work was supported by National Key Research and Development Program of China (2018YFA0702100), and Key Research and Development Program of Zhejiang Province (2021C05002).

\section{Conflicts of interest}

All authors declared that there are no conflicts of interest.

\section{Ethical approval and consent to participate}

Not applicable.

\section{Consent for publication}

Not applicable.

\section{Copyright}

(c) The Author(s) 2021. 


\section{REFERENCES}

1. $\quad \mathrm{Yu} \mathrm{H}, \mathrm{Li}$ N, Zhao N. How far are we from achieving self $\square$ powered flexible health monitoring systems: an energy perspective. $A d v$ Energy Mater 2021;11:2002646. DOI

2. Wu C, Kim TW, Park JH, et al. Self-powered tactile sensor with learning and memory. ACS Nano 2020;14:1390-8. DOI PubMed

3. Yin R, Wang D, Zhao S, Lou Z, Shen G. Wearable sensors - enabled human-machine interaction systems: from design to application. Adv Funct Mater 2021;31:2008936. DOI

4. Wu Y, Liu Y, Zhou Y, et al. A skin-inspired tactile sensor for smart prosthetics. Sci Robot 2018;3:eaat0429. DOI PubMed

5. Pyo S, Lee J, Bae K, Sim S, Kim J. Recent progress in flexible tactile sensors for human-interactive systems: from sensors to advanced applications. Adv Mater 2021:e2005902. DOI PubMed

6. Shi J, Wang L, Dai Z, et al. Multiscale hierarchical design of a flexible piezoresistive pressure sensor with high sensitivity and wide linearity range. Small 2018;14:e1800819. DOI PubMed

7. Yamamoto Y, Harada S, Yamamoto D, et al. Printed multifunctional flexible device with an integrated motion sensor for health care monitoring. Sci $A d v$ 2016;2:e1601473. DOI PubMed PMC

8. Dahiya RS, Valle M. Robotic tactile sensing: technologies and system. Springer: Science \& Business Media; 2012.

9. Sundaram S, Kellnhofer P, Li Y, Zhu JY, Torralba A, Matusik W. Learning the signatures of the human grasp using a scalable tactile glove. Nature 2019;569:698-702. DOI PubMed

10. Wang Z, Guan X, Huang H, Wang H, Lin W, Peng Z. Full 3D printing of stretchable piezoresistive sensor with hierarchical porosity and multimodulus architecture. Adv Funct Mater 2019;29:1807569. DOI

11. Park YJ, Sharma BK, Shinde SM, et al. All $\mathrm{MoS}_{2}$-based large area, skin-attachable active-matrix tactile sensor. ACS Nano 2019;13:3023-30. DOI PubMed

12. Ma L, Shuai X, Hu Y, et al. A highly sensitive and flexible capacitive pressure sensor based on a micro-arrayed polydimethylsiloxane dielectric layer. J Mater Chem C 2018;6:13232-40. DOI

13. Chen X, Shao J, Tian H, et al. Scalable imprinting of flexible multiplexed sensor arrays with distributed piezoelectricity - enhanced micropillars for dynamic tactile sensing. Adv Mater Technol 2020;5:2000046. DOI

14. Chen Z, Wang Z, Li X, et al. Flexible piezoelectric-induced pressure sensors for static measurements based on nanowires/graphene heterostructures. ACS Nano 2017;11:4507-13. DOI PubMed

15. Wang X, Zhang H, Dong L, et al. Self-powered high-resolution and pressure-sensitive triboelectric sensor matrix for real-time tactile mapping. Adv Mater 2016;28:2896-903. DOI PubMed

16. Rao J, Chen Z, Zhao D, et al. Tactile electronic skin to simultaneously detect and distinguish between temperature and pressure based on a triboelectric nanogenerator. Nano Energy 2020;75:105073. DOI

17. Shin J, Jeong B, Kim J, et al. Sensitive wearable temperature sensor with seamless monolithic integration. Adv Mater 2020;32:e1905527. DOI PubMed

18. Dinh T, Phan H, Qamar A, Woodfield P, Nguyen N, Dao DV. Thermoresistive effect for advanced thermal sensors: fundamentals, design considerations, and applications. J Microelectromech Syst 2017;26:966-86. DOI

19. Li M, Chen J, Zhong W, et al. Large-area, wearable, self-powered pressure-temperature sensor based on 3D thermoelectric spacer fabric. ACS Sens 2020;5:2545-54. DOI PubMed

20. Horta Romarís L, González Rodríguez MV, Huang B, et al. Multifunctional electromechanical and thermoelectric polyanilinepoly(vinyl acetate) latex composites for wearable devices. J Mater Chem C 2018;6:8502-12. DOI

21. Lee M, Nicholls H. Review Article Tactile sensing for mechatronics - a state of the art survey. Mechatronics 1999;9:1-31. DOI

22. Lee S, Reuveny A, Reeder J, et al. A transparent bending-insensitive pressure sensor. Nat Nanotechnol 2016;11:472-8. DOI PubMed

23. Wu C, Wang AC, Ding W, Guo H, Wang ZL. Triboelectric nanogenerator: a foundation of the energy for the new era. Adv Energy Mater 2019;9:1802906. DOI

24. Liu D, Shi P, Ren W, et al. A new kind of thermocouple made of p-type and n-type semi-conductive oxides with giant thermoelectric voltage for high temperature sensing. J Mater Chem C 2018;6:3206-11. DOI

25. Shi L, Li Z, Chen M, Qin Y, Jiang Y, Wu L. Quantum effect-based flexible and transparent pressure sensors with ultrahigh sensitivity and sensing density. Nat Commun 2020;11:3529. DOI PubMed PMC

26. Yang JC, Mun J, Kwon SY, Park S, Bao Z, Park S. Electronic skin: recent progress and future prospects for skin-attachable devices for health monitoring, robotics, and prosthetics. Adv Mater 2019;31:e1904765. DOI PubMed

27. Bae GY, Pak SW, Kim D, et al. Linearly and highly pressure-sensitive electronic skin based on a bioinspired hierarchical structural array. Adv Mater 2016;28:5300-6. DOI PubMed

28. Chou HH, Nguyen A, Chortos A, et al. A chameleon-inspired stretchable electronic skin with interactive colour changing controlled by tactile sensing. Nat Commun 2015;6:8011. DOI PubMed PMC

29. Zhao XH, Ma SN, Long H, et al. Multifunctional sensor based on porous carbon derived from metal-organic frameworks for real time health monitoring. ACS Appl Mater Interfaces 2018;10:3986-93. DOI PubMed

30. Wang C, Xia K, Zhang M, Jian M, Zhang Y. An all-silk-derived dual-mode E-skin for simultaneous temperature-pressure detection. ACS Appl Mater Interfaces 2017;9:39484-92. DOI PubMed

31. Zu G, Kanamori K, Nakanishi K, Huang J. Superhydrophobic ultraflexible triple-network graphene/polyorganosiloxane aerogels for a high-performance multifunctional temperature/strain/pressure sensing array. Chem Mater 2019;31:6276-85. DOI

32. Zhang F, Zang Y, Huang D, Di CA, Zhu D. Flexible and self-powered temperature-pressure dual-parameter sensors using microstructure-frame-supported organic thermoelectric materials. Nat Commun 2015;6:8356. DOI PubMed PMC 
33. Han S, Jiao F, Khan ZU, Edberg J, Fabiano S, Crispin X. Thermoelectric polymer aerogels for pressure-temperature sensing applications. Adv Funct Mater 2017;27:1703549. DOI

34. Wang Y, Wu H, Xu L, Zhang H, Yang Y, Wang ZL. Hierarchically patterned self-powered sensors for multifunctional tactile sensing. Sci Adv 2020;6:eabb9083. DOI PubMed PMC

35. Wang Y, Mao H, Wang Y, Zhu P, Liu C, Deng Y. 3D geometrically structured PANI/CNT-decorated polydimethylsiloxane active pressure and temperature dual-parameter sensors for man-machine interaction applications. J Mater Chem A 2020;8:15167-76. DOI

36. Gui Q, He Y, Gao N, Tao X, Wang Y. A skin-inspired integrated sensor for synchronous monitoring of multiparameter signals. $A d v$ Funct Mater 2017;27:1702050. DOI

37. Jung M, Vishwanath SK, Kim J, et al. Transparent and flexible mayan-pyramid-based pressure sensor using facile-transferred indium tin oxide for bimodal sensor applications. Sci Rep 2019;9:14040. DOI PubMed PMC

38. Wang Z, Zhang L, Liu J, Li C. A flexible bimodal sensor based on an electrospun nanofibrous structure for simultaneous pressuretemperature detection. Nanoscale 2019;11:14242-9. DOI PubMed

39. Bae GY, Han JT, Lee G, et al. Pressure/temperature sensing bimodal electronic skin with stimulus discriminability and linear sensitivity. Adv Mater 2018;30:e1803388. DOI PubMed

40. Wu R, Ma L, Hou C, et al. Silk composite electronic textile sensor for high space precision 2D combo temperature-pressure sensing. Small 2019;15:e1901558. DOI PubMed

41. Shin S, Lee W, Kim S, et al. Ion-conductive self-healing hydrogels based on an interpenetrating polymer network for a multimodal sensor. Chem Eng J 2019;371:452-60. DOI

42. Jung M, Kim K, Kim B, et al. Paper-Based Bimodal Sensor for Electronic Skin Applications. ACS Appl Mater Interfaces 2017;9:26974-82. DOI PubMed

43. Jung M, Kim K, Kim B, Lee KJ, Kang JW, Jeon S. Vertically stacked nanocellulose tactile sensor. Nanoscale 2017;9:17212-9. DOI PubMed

44. Kim K, Jung M, Kim B, et al. Low-voltage, high-sensitivity and high-reliability bimodal sensor array with fully inkjet-printed flexible conducting electrode for low power consumption electronic skin. Nano Energy 2017;41:301-7. DOI

45. Zhu P, Wang Y, Sheng M, Wang Y, Yu Y, Deng Y. A flexible active dual-parameter sensor for sensitive temperature and physiological signal monitoring via integrating thermoelectric and piezoelectric conversion. J Mater Chem A 2019;7:8258-67. DOI

46. Zhu P, Wang Y, Wang Y, Mao H, Zhang Q, Deng Y. Flexible 3D architectured piezo/thermoelectric bimodal tactile sensor array for E-skin application. Adv Energy Mater 2020;10:2001945. DOI

47. Song K, Zhao R, Wang ZL, Yang Y. Conjuncted pyro-piezoelectric effect for self-powered simultaneous temperature and pressure sensing. Adv Mater 2019;31:e1902831. DOI PubMed

48. Yu J, Kiwi J, Zivkovic I, Rønnow HM, Wang T, Rtimi S. Quantification of the local magnetized nanotube domains accelerating the photocatalytic removal of the emerging pollutant tetracycline. Appl Catal B 2019;248:450-8. DOI

49. O'regan B, Grätzel M. A low-cost, high-efficiency solar cell based on dye-sensitized colloidal TiO2 films. Nature 1991;353:737-40. DOI

50. Michaels H, Rinderle M, Freitag R, et al. Dye-sensitized solar cells under ambient light powering machine learning: towards autonomous smart sensors for the internet of things. Chem Sci 2020;11:2895-906. DOI PubMed PMC

51. Wang Y, Zhu W, Deng Y, et al. Self-powered wearable pressure sensing system for continuous healthcare monitoring enabled by flexible thin-film thermoelectric generator. Nano Energy 2020;73:104773. DOI

52. Wang Y, Liu G, Sheng M, Yu C, Deng Y. Flexible thermopower generation over broad temperature range by PANI/nanorod hybridbased p-n couples. J Mater Chem A 2019;7:1718-24. DOI

53. Feng J, Zhu W, Zhang Z, Cao L, Yu Y, Deng Y. Enhanced electrical transport properties via defect control for screen-printed $\mathrm{Bi}_{2} \mathrm{Te}_{3}$ films over a wide temperature range. ACS Appl Mater Interfaces 2020;12:16630-8. DOI PubMed

54. Sheng M, Wang Y, Liu C, Xiao Y, Zhu P, Deng Y. Significantly enhanced thermoelectric performance in SWCNT films via carrier tuning for high power generation. Carbon 2020;158:802-7. DOI

55. Park DY, Joe DJ, Kim DH, et al. Self-Powered real-time arterial pulse monitoring using ultrathin epidermal piezoelectric sensors. Adv Mater 2017;29:1702308. DOI PubMed

56. Chen X, Li X, Shao J, et al. High-performance piezoelectric nanogenerators with imprinted P(VDF-TrFE)/BaTiO ${ }_{3}$ nanocomposite micropillars for self-powered flexible sensors. Small 2017;13:1604245. DOI PubMed

57. Fan FR, Lin L, Zhu G, Wu W, Zhang R, Wang ZL. Transparent triboelectric nanogenerators and self-powered pressure sensors based on micropatterned plastic films. Nano Lett 2012;12:3109-14. DOI PubMed

58. Varma S, Sambath Kumar K, Seal S, Rajaraman S, Thomas J. Fiber-type solar cells, nanogenerators, batteries, and supercapacitors for wearable applications. Adv Sci (Weinh) 2018;5:1800340. DOI PubMed PMC

59. Pan Z, Ren J, Guan G, et al. Synthesizing nitrogen-doped core-sheath carbon nanotube films for flexible lithium ion batteries. $A d v$ Energy Mater 2016;6:1600271. DOI

60. Wang N, Dou W, Hao S, et al. Tactile sensor from self-chargeable piezoelectric supercapacitor. Nano Energy 2019;56:868-74. DOI

61. Wang J, Lou H, Meng J, Peng Z, Wang B, Wan J. Stretchable energy storage E-skin supercapacitors and body movement sensors. Sens Actuators B Chem 2020;305:127529. DOI

62. Han S, Kim J, Won SM, et al. Battery-free, wireless sensors for full-body pressure and temperature mapping. Sci Transl Med 2018;10:eaan4950. DOI PubMed PMC

63. Song Y, Min J, Yu Y, et al. Wireless battery-free wearable sweat sensor powered by human motion. Sci Adv 2020;6:eaay9842. DOI PubMed PMC 
64. Luo Y, Li Y, Sharma P, et al. Learning human-environment interactions using conformal tactile textiles. Nat Electron 2021;4:193-201. DOI

65. An BW, Heo S, Ji S, Bien F, Park JU. Transparent and flexible fingerprint sensor array with multiplexed detection of tactile pressure and skin temperature. Nat Commun 2018;9:2458. DOI PubMed PMC 